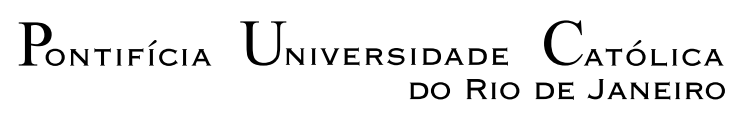
DO RIO DE JANEIRO

Breno Somazz

\title{
Análise Modal de Sistemas Mecânicos
}

\section{Projeto de Graduação}

Projeto de Graduação apresentado ao Departamento de Engenharia Mecânica da PUC-Rio.

Orientador : Roberta Lima

Coorientador: Rubens Sampaio 


\section{Agradecimentos}

Agradeço a minha orientadora e ao meu coorientador pelo incentivo e orientação ao desenvolvimento deste trabalho. 


\section{Resumo}

\section{Análise Modal de Sistemas Mecânicos}

O trabalho desenvolvido começa apresentando os principais conceitos relacionados a dinâmica de sistemas mecânicos, com um e com múltiplos graus de liberdade, tendo como objetivo a modelagem analítica e a simulação numérica de tais sistemas, a fim de entender como que estes se comportam sob a ação de esforços externos.

O trabalho concentra-se no método denominado decomposição modal, que consiste na determinação dos parâmetros modais (frequências naturais e modos de vibração) e, a partir destes, decompor a dinâmica do sistema, com o objetivo de determinar como que tais parâmetros a influenciam. A ferramenta que é utilizada para realizar este procedimento, é a matriz de função de reposta em frequência $(F R F)$ do sistema, cujos elementos são definidos em função dos parâmetros modais. A partir do conhecimendo dos elementos da matriz de FRF, pode-se determinar como que um sistema mecânico se comporta quando sujeito a esforços externos.

Os tópicos relacionados a teoria de análise modal são aplicados a um sistema mecânico com cinco graus de liberdade, cujo estudo é dividido em dois casos: sem amortecimento e com amortecimento linear viscoso. Ambos os casos foram simulados com o uso do software MatLab, nos domínios do tempo e da frequência.

A partir dos resultados obtidos, pode-se verificar os principais conceitos relacionados a teoria de análise modal e, a partir destes, entender de que forma um sistema mecânico se comporta sob carregamentos externos.

\section{Palavras-chave}

Dinâmica; Vibrações; Função de resposta em frequência; Frequências naturais; Modos de vibração; Ressonância. 


\section{Abstract}

\section{Modal Analysis of Mechanical Systems}

The work begins by presenting the main concepts related to the dynamics of mechanical systems, with one and multiple degrees of freedom, aiming at the analytical modeling and numerical simulation of such systems, in order to understand how they behave under the action of external forces.

The work focuses on a method called modal decomposition, which consists in determining the modal parameters (natural frequencies and vibration modes) of a system and in decomposition of the dynamics using these modal parameters. The objective is to determine how these parameters influence it. A tool that is used to perform this procedure is the frequency response function (FRF) matrix of the system, whose elements are denoted as a function of modal parameters. From the knowledge of the elements of the FRF matrix, one can determine how a mechanical system behaves when subjected to external forces.

The topics related to the theory of modal analysis are applied to a mechanical system with five degrees of freedom, whose study is divided into two cases: without damping and with linear viscous damping. Both cases were simulated using MatLab software, in time and frequency domains.

From the results obtained, it is possible to verify the main concepts related to the theory of modal analysis and, to understand how a mechanical system behaves under external loads.

\section{Keywords}

Dynamics; Vibrations; Frequency response function; Natural frequencies; Vibration modes; Ressonance. 


\section{Sumário}

\begin{tabular}{lll}
\hline 1 & Introdução & 7
\end{tabular}

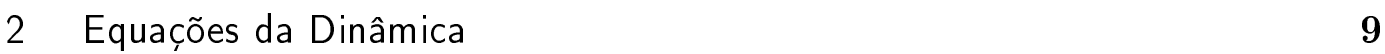

3 Sistemas com um Grau de Liberdade $\quad 12$

3.1 Sistemas com Amortecimento Linear Viscoso 12

$\begin{array}{lll}3.2 & \text { Sistemas com Amortecimento Estrutural } & 15\end{array}$

4 Sistemas com Múltiplos Graus de Liberdade 18

$\begin{array}{lll}4.1 & \text { Sistemas sem Amortecimento } & 18\end{array}$

4.2 Sistemas com Amortecimento 23

5 Modelagem Analítica $\quad 32$

$\begin{array}{lll}5.1 & \text { Sistema 1 } & 32\end{array}$

$\begin{array}{lll}5.2 & \text { Sistema 2 } & 35\end{array}$

$6 \quad$ Simulação Numérica e Análise dos Resultados 39

\begin{tabular}{lll}
\hline 6.1 & Sistema 1 & 39
\end{tabular}

6.2 Sistema 2 sem Amortecimento 43

6.3 Sistema 2 com Amortecimento 48

\begin{tabular}{lll}
\hline 7 & Conclusão & 53
\end{tabular} 


\section{Lista de figuras}

$\begin{array}{lll}5.1 & \text { sistema } 1 . & 32\end{array}$

5.2 sistema 2. 35

6.1 resposta no tempo do sistema 1.

6.2 gráfico de Bode do sistema 1.

6.3 gráfico real e imaginário do sistema 1.

6.4 gráfico de Nyquist do sistema 1.

6.5 amplitude da resposta em regime permanente do sistema 1.

6.6 amplitude da resposta em regime permanente do sistema 1, para diferentes valores de $\zeta$. 42

6.7 resposta no tempo do sistema $2 \mathrm{sem}$ amortecimento, para $\omega_{f}=$ $5 \mathrm{rad} / \mathrm{s}$.

6.8 resposta no tempo do sistema 2 sem amortecimento, para $\omega_{f}=\omega_{1}$. 46

\begin{tabular}{|ll|l}
\hline 6.9 & resposta em frequência do sistema 2 sem amortecimento. & 46
\end{tabular}

6.10 resposta em frequência do sistema 2 sem amortecimento. 47

6.11 resposta em frequência do sistema 2 sem amortecimento. $\quad 48$

6.12 resposta no tempo do sistema 2 com amortecimento para $\omega_{f}=$

6.13 resposta em frequência do sistema 2 com amortecimento. 51

6.14 resposta em frequência do sistema 2 com amortecimento. 52 


\section{Introdução}

A dinâmica de um sistema mecânico é governada por um problema que depende da forma como o sistema é modelado. Se este for modelado como discreto, apresentará um ou múltiplos graus de liberdade, se for modelado como contínuo apresentará infinitos graus de liberdade.

O número de graus de liberdade de um sistema mecânico é o número mínimo de coordenadas que são necessárias para se descrever completamente o movimento do sistema para todo instante de tempo. Para um sistema mecânico discreto com um grau de liberdade, ou seja, cuja posição ao longo do tempo é descrita por uma única coordenada (por exemplo, um sistema massa-molaamortecedor), a dinâmica será dada por um problema de valor inicial (P.V.I), consistindo neste caso de uma equação diferencial ordinária (e.d.o) de segunda ordem e duas condições iniciais (C.I's). Quando existem múltiplos graus de liberdade, será necessário um número $n$ de e.d.o's de segunda ordem, cada uma com duas C.I's associadas. No caso do sistema ser modelado como contínuo, a dinâmica deste será dada por um problema de valor de contorno (P.V.C), que será composto por uma única equação diferencial parcial (e.d.p), C.I's e condições de contorno (C.C's).

No presente trabalho, os sistemas mecânicos serão modelados como discretos com múltiplos graus de liberdade. Além disto, serão utilizadas hipóteses simplificadoras que permitam obter e.d.o's lineares de segunda ordem para descrever a dinâmica dos sistemas mecânicos tratados.

Um sistema mecânico pode ser analisado no domínio do tempo ou no domínio da frequência. Analisá-lo no domínio do tempo significa conhecer a sua dinâmica e consequentemente prever o seu comportamento ao longo do tempo a partir de uma dada C.I. Quando este é analisado no domínio da frequência, significa conhecer o efeito da excitação externa no seu comportamento dinâmico, ou seja, determinar de que forma o sistema irá se comportar dado que carregamentos externos são aplicados sobre ele. O que permite realizar a análise no domínio da frequência, para o caso de sistemas com um grau de liberdade, é a função de resposta em frequência (FRF), e para sistemas com múltiplos graus de liberdade é a matriz de FRF. De acordo com [1], [2] e [3], a FRF para o caso de um sistema com um grau de liberdade é a relação entre a amplitude do forçamento externo e a amplitude da resposta do sistema em regime permanente, dada em função da frequência do forçamento externo.

Quando o sistema tem múltiplos graus de liberdade, de acordo com [1] 
e [3], os elementos $h_{i j}(\omega)$ da matriz de FRF expressam a relação entre a amplitude do forçamento externo que age no grau de liberdade $j$ e a amplitude da resposta do sistema em regime permanente referente ao grau de liberdade $i$, caso haja um único forçamento atuando no sistema e este esteja agindo no grau de liberdade $j$.

A análise modal de sistemas mecânicos consiste na determinação da matriz de FRF do sistema. Esta pode ser obtida de forma experimental ou através da modelagem analítica do sistema. O presente trabalho tem como objetivo a determinação da matriz de FRF de sistemas mecânicos de forma analítica. Primeiramente, será apresentada a teoria necessária a ser utilizada na modelagem analítica dos sistemas e, em seguida, os sistemas mecânicos serão definidos e modelados analíticamente. Ao final, será feita a simulação numérica dos sistemas utilizando-se o software MatLab e os resultados obtidos serão discutidos. 


\section{2}

\section{Equações da Dinâmica}

Existem diferentes formas de se encontrar as equações diferenciais que determinam a dinâmica de um sistema mecânico. Há dois métodos muito utilizados para se obtê-las. O primeiro é aquele que utiliza diretamente a segunda lei de Newton, e que precisa, necessariamente, da determinação de todas as forças que atuam em cada parte do sistema. Este método é vantajoso quando o sistema possui um número pequeno de graus de liberdade, de forma que os diagramas de corpo livre de cada uma das massas (ou corpos rígidos) que compõem o sistema possam ser representados e as forças sejam simples de serem identificadas. Porém, quando o sistema mecânico tratado apresenta um número maior de graus de liberdade, este método se torna complicado, pois será necessário um número muito maior de diagramas de corpo livre e as interações entre cada uma das partes do sistema se tornarão mais complexas, o que dificulta a identificação das forças que atuam em cada uma destas.

Como alternativa para este problema, existe o método de Lagrange, que permite modelar a dinâmica baseando-se na energia do sistema. A vantagem de se utilizar este método é que as equações obtidas já se encontram em uma forma escalar, não sendo necessária a identificação de todas as forças que atuam em cada parte do sistema, bem como desenhar diagramas de corpo livre. Além disso, este método permite a modelagem de sistemas com múltiplos graus de liberdade de uma forma mais direta e objetiva, seguindo-se uma formulação que é única e que pode ser aplicada a uma variedade de sistemas mecânicos. No presente trabalho, esta será a metodologia utilizada para se modelar a dinâmica dos sistemas mecânicos que serão tratados mais adiante.

O primeiro passo para aplicar o método de Lagrange é definir o Lagrangeano $L$ do sistema, dado pela diferença entre a energia cinética e potencial do sistema, ou seja,

$$
L=T-V,
$$

onde $T$ e $V$ são, respectivamente, as energias cinética e potencial total do sistema. O termo de energia cinética $T$, é a soma de todas as energias cinéticas referentes a cada massa ou corpo rígido utilizado para modelar o sistema mecânico real. Da mesma forma, o termo de energia potencial $V$ é dado pela soma de todas as energias potenciais referentes a cada massa ou corpo rígido, 
bem como de cada mola utilizada para modelar o efeito de armazenamento de energia do sistema.

Após ter calculado a expressão do Lagrangeano, esta será uma função das várias coordenadas que representam cada um dos graus de liberdade do sistema. Considerando-se o caso em que não existam forças dissipativas no sistema, ou seja, quando não há nenhuma força de atrito ou força externa não conservativa atuando no sistema, as equações da dinâmica para cada um dos graus de liberdade do sistema são obtidas a partir da equação de EulerLagrange, dada por

$$
\frac{d}{d t}\left(\frac{\partial L}{\partial \dot{x}_{i}}\right)-\frac{\partial L}{\partial x_{i}}=0
$$

onde $x_{i}, i=1, \ldots, n$ é a coordenada que representa o i-ésimo grau de liberdade do sistema. Para o caso de atuarem forças de atrito ou forças externas não conservativas no sistema, a equação (2-2) sofrerá uma modificação a fim de englobar o efeito dessas forças na dinâmica do sistema.

De acordo com [2], há dois conceitos importantes, o conceito de coordenadas generalizadas e o trabalho virtual. Conforme apresentado em [2, a dinâmica de um sistema mecânico pode ser representada por diferentes conjuntos de coordenadas, que expressam as diferentes formas de se parametrizar um determinado sistema, ou seja, de escolher quais coordenadas representarão o seu movimento. Segundo [2], quando existir uma relação de dependência entre as coordenadas de um mesmo conjunto, tais relações são denominadas equações de vínculo. Ainda de acordo com este, quando as coordenadas escolhidas para parametrizar o movimento do sistema são independentes entre si, estas são denominadas de coordenadas generalizadas.

Segundo [2], o trabalho virtual é o trabalho realizado por uma força e que produzirá um deslocamento virtual em uma coordenada generalizada. Este deslocamento virtual, de acordo com [2], é, por definição, um deslocamento infinitesimal, que não deve alterar as equações de vínculo nem alterar a geometria do sistema. A força que produzirá o trabalho virtual é, de acordo com [2], denominada força generalizada e é definida por

$$
Q_{i}=\frac{\delta W}{\delta q_{i}}
$$

onde $Q_{i}$ é a força generalizada que produzirá o trabalho virtual $\delta W$, que causará o deslocamento virtual $\delta q_{i}$ na i-ésima coordenada generalizada $q_{i}$ do 
sistema, se esta representar um movimento de translação. De acordo com [2], se $q_{i}$ representar um movimento de rotação, $Q_{i}$ será um momento.

Desta forma, se os graus de liberdade de um sistema mecânico forem todos representados por coordenadas generalizadas, e se neste sistema estiverem atuando forças de atrito e forças externas não conservativas, as equações da dinâmica do sistema, que representam a evolução temporal dos graus de liberdade, serão dadas por

$$
\frac{d}{d t}\left(\frac{\partial L}{\partial \dot{x}_{i}}\right)-\frac{\partial L}{\partial x_{i}}=Q_{i}
$$

onde $x_{i}, i=1, \ldots, n$ é a coordenada generalizada que representa o i-ésimo grau de liberdade do sistema e $Q_{i}$ é a i-ésima força de atrito ou força externa não conservativa que atua no sistema no i-ésimo grau de liberdade.

Para o caso de existirem forças de atrito do tipo linear viscoso, a equação (2-4) recebe mais um termo, sendo reescrita como

$$
\frac{d}{d t}\left(\frac{\partial L}{\partial \dot{x}_{i}}\right)-\frac{\partial L}{\partial x_{i}}=Q_{i}-\frac{\partial D}{\partial \dot{x}_{i}}
$$

onde, de acordo com [4], $D=\frac{1}{2} c \dot{x}_{i}^{2}$ é a função dissipação de Rayleigh e $c$ é o coeficiente de atrito linear viscoso. 


\section{Sistemas com um Grau de Liberdade}

\section{1}

\section{Sistemas com Amortecimento Linear Viscoso}

Sistemas mecânicos com um grau de liberdade tem sua dinâmica governada por um P.V.I. No caso do sistema ser modelado como linear e invariante no tempo (SLIT), a e.d.o que representa a dinâmica será linear de segunda ordem com coeficientes constantes, tendo a seguinte forma geral

$$
m \ddot{x}(t)+c \dot{x}(t)+k x(t)=u(t)
$$

que é a e.d.o de um sistema massa-mola-amortecedor com coeficiente de amortecimento linear viscoso $c$ e uma mola linear de rigidez $k$, sujeito a um forçamento externo $u(t)$. A função $x(t)$ representa o único grau de liberdade do sistema, ou seja, a posição da massa $m$ ao longo do tempo, medida a partir da posição em que a mola encontra-se relaxada.

Neste caso, de acordo com [3], podemos considerar tais sistemas como filtros, ou seja, dada uma entrada (forçamento externo), obtêm-se como saída a resposta do sistema (posição ao longo do tempo) através de uma operação realizada sobre a entrada.

Desta forma, analisando-se o sistema no domínio do tempo, dada uma entrada $u(t)$, a resposta do sistema, considerando C.I's nulas de posição e velocidade, de acordo com [3], será dada por

$$
x(t)=\int_{0}^{t} h(t-\tau) u(\tau) d \tau,
$$

onde $h(t)$ é a resposta do sistema ao impulso unitário.

O impulso unitário, segundo [3], é uma representação de uma força que é aplicada instantâneamente ao sistema. Fisicamente isto não é possível, tratase apenas de uma definição matemática que permite o cálculo da resposta do sistema a partir de uma entrada conhecida. A definição do impulso unitário mostrada em [3] é dada por 


$$
\delta(t)= \begin{cases}+\infty, & t=0 \\ 0, & t \neq 0\end{cases}
$$

Esta definição afirma que no instante inicial a força aplicada tem módulo infinito e nos demais ela é nula. A partir desta definição, conforme mostrado em [3], $h(t)$ é a resposta livre do sistema (sem forçamento externo, ou seja $u(t)=0)$ com C.I's $x(0)=0$ e $\dot{x}(0)=1 / m$.

A integral dada da equação (3-2) é uma operação matemática conhecida como convolução, e é denotada pelo simbolo *. Portanto, a resposta do sistema no domínio do tempo, com C.I's nulas, é dada por $x(t)=h(t) * u(t)$. Esta forma de calcular a resposta do sistema, através da operação da convolução, permite tratar diferentes tipos de entradas e torna a abordagem do problema mais direta, pois conhecida a entrada que está sendo aplicada ao sistema, basta calcular a resposta ao impulso unitário e em seguida realizar a convolução entre ambas para se obter a resposta do sistema.

No entanto, dependendo do tipo de forçamento externo que é aplicado ao sistema, a operação da convolução pode se tornar trabalhosa. Neste caso, um outro método, denominado método dos coeficientes indeterminados, pode ser utilizado para se obter a resposta do sistema no domínio do tempo. Este método considera que

$$
x(t)=x_{H}(t)+x_{p}(t),
$$

onde $x_{H}(t)$ é a solução da equação homogênea (caso em que $u(t)=0$ ) associada a equação (3-1) e $x_{p}(t)$ é uma solução particular da equação (3-1) (equação não homogênea).

A análise no domínio da frequência é feita de forma semelhante, ou seja, considerando ainda o sistema como um filtro, irá se obter a resposta através de uma operação realizada sobre a entrada. Neste caso, a resposta do sistema no domínio da frequência será dada por

$$
X(\omega)=H(\omega) \cdot U(\omega)
$$

onde $H(\omega)$ e $U(\omega)$ são, respectivamente, a transformada de Fourier da resposta ao impulso unitário $h(t)$ e da entrada $u(t)$. A função $H(\omega)$ é a FRF do sistema. Assim como no caso do domínio do tempo, no domínio da frequência 
deve-se conhecer a entrada e realizar uma operação entre esta e a resposta ao impulso unitário, porém agora considerando ambas no domínio da frequência, sendo que neste caso, a convolução dada por uma integral no domínio do tempo, se torna um produto entre funções no domínio da frequência.

Há também uma outra forma de se obter a FRF para um sistema com um grau de liberdade. Esta forma de se calcular $H(\omega)$ é mostrada em [2] e em [3. Supondo que um forçamento harmônico externo $f(t)=F_{0} \cos (\omega t)$ esteja agindo sobre a massa $m$ do sistema, a equação (3-1) se torna

$$
m \ddot{x}(t)+c \dot{x}(t)+k x(t)=F_{0} \cos (\omega t) .
$$

Conforme mostrado em [2], pode-se reescrever o forçamento externo usando-se a equação de Euler $A e^{j \omega t}=A \cos (\omega t)+A \operatorname{sen}(\omega t) j$, onde $j=\sqrt{-1}$ é a unidade imaginária. Desta forma, substituindo o forçamento externo por $f(t)=F_{0} e^{j \omega t}$, a equação (3-6) se torna

$$
m \ddot{X}(t)+c \dot{X}(t)+k X(t)=F_{0} e^{j \omega t},
$$

que segundo [2], terá como solução particular uma função complexa $X_{p}(t)=$ $X_{0} e^{j \omega t}$ cuja parte real é a solução física $x(t)$ original. Substituindo $X_{p}(t)$ e suas derivadas na equação (3-7) e rearranjando os termos, obtêm-se

$$
\left(-m \omega^{2}+c \omega j+k\right) X_{0} e^{j \omega t}=F_{0} e^{j \omega t} .
$$

Dividindo ambos os lados da equação (3-8) por $e^{j \omega t}$ e rearranjando os termos, obtêm-se

$$
\frac{X_{0}}{F_{0}}=\frac{1}{\left(-m \omega^{2}+c \omega j+k\right)} .
$$

Conforme definido em [3], o termo $\left(-m \omega^{2}+c \omega j+k\right)$ é denominado rigidez dinâmica do sistema e denotado por $K_{d i n}$, e o seu inverso é a FRF do sistema. Desta forma, tem-se que

$$
H(\omega)=\frac{1}{\left(-m \omega^{2}+c \omega j+k\right)} .
$$


Mais especificamente, como descrito em [3], $H(\omega)$ é a FRF do deslocamento, e pode também ser obtida para as grandezas velocidade e aceleração, basta multiplicar ambos os lados da equação (3-10) por $j \omega$ ou por $-\omega^{2}$ para se obter, respectivamente, as FRF's da velocidade e da aceleração, dadas por $H_{v}=j \omega H(\omega)$ e $H_{a}=-\omega^{2} H(\omega)$.

Também é possível, de acordo com [3, escrever a FRF em função da razão de frequências $r=\frac{\omega}{\omega_{n}}$ e da taxa de amortecimento $\zeta=\frac{c}{2 m \omega_{n}}$, onde $\omega$ é a frequência do forçamento externo e $\omega_{n}=\sqrt{\frac{k}{m}}$ é a frequência natural do sistema conservativo associado (que representa o caso em que $c=0$ ). Desta forma, a FRF é escrita como

$$
H(\omega)=\frac{1}{k\left(1-r^{2}+2 \zeta r j\right)} .
$$

A representação gráfica de $H(\omega)$ não pode ser feita de uma única forma, pois esta é uma função complexa, e portanto, seu gráfico, como mostrado em [1], é dado por uma superfície contida em um espaço tridimensional, representado por três eixos perpendiculares entre si: um eixo real, um imaginário e um de frequências. Desta forma, a análise gráfica da FRF é feita a partir da projeção do seu gráfico tridimensional em cada um dos planos formados por dois desses eixos.

Cada uma das curvas obtidas a partir da projeção do gráfico de $H(\omega)$ em cada um dos planos recebe um nome especial, e revela características diferentes da FRF. Como mostrado em [1] e [3], o gráfico de Bode é composto por dois gráficos: o gráfico da magnitude de $H(\omega)$ versus a frequência e o gráfico da fase de $H(\omega)$ versus a frequência. O gráfico real e o gráfico imaginário são dados, respectivamente, pela parte real e imaginária de $H(\omega)$ versus a frequência. $\mathrm{O}$ gráfico de Nyquist é dado pela parte imaginária versus a parte real de $H(\omega)$.

\section{2}

\section{Sistemas com Amortecimento Estrutural}

Utilizar o amortecimento linear viscoso na modelagem de um sistema com um grau de liberdade não é a única forma de se tratar o amortecimento presente no sistema mecânico real considerado, pois além do amortecimento linear viscoso, existem diversos outros modelos que são conhecidos e que podem ser utilizados para modelar as forças de atrito presentes no sistema. Um destes modelos, que é apresentado em [1] e [2], é o amortecimento estrutural.

Conforme explicado em [2], o amortecimento estrutural está presente 
quando um componente mecânico sofre ciclos de carregamento e descarregamento, de forma que a deformação causada por estes ciclos irá fazer com que as partes internas do componente sofram atrito entre si, a medida que o componente é deformado sob tração ou compressão. Como mostrado em [2], um sistema com este tipo de amortecimento pode ser modelado por uma e.d.o semelhante aquela obtida para um sistema modelado com amortecimento linear viscoso.

Para se obter a equação da dinâmica para um sistema de um grau de liberdade cujas forças de atrito são modeladas como sendo de amortecimento estrutural, deve-se, de acordo com [2], obter um coeficiente de amortecimento linear viscoso equivalente para o amortecimento estrutural. A partir deste coeficiente equivalente, conforme mostrado em [2], chega-se a uma e.d.o para a dinâmica do sistema mecânico com amortecimento estrutural.

Como mostrado em [2], isto é feito fazendo uma equivalência entre a energia dissipada por ciclo por um amortecedor com amortecimento linear viscoso sob um carregamento harmônico e a energia dissipada por ciclo devido ao amortecimento estrutural. De acordo com [2], a energia dissipada por ciclo por um amortecedor com amortecimento linear viscoso, após o sistema já ter atingido o regime permanente, é dada por

$$
\Delta E=\pi c \omega X^{2}
$$

onde $c$ é o coeficiente de amortecimento linear viscoso, $\omega$ é a frequência do carregamento externo e $X$ a amplitude da resposta do sistema em regime permanente. De acordo com [2], obtêm-se experimentalmente que a energia dissipada por ciclo por um componente mecânico com amortecimento estrutural é dada por

$$
\Delta E=\pi k \beta X^{2}
$$

onde $k$ é a rigidez do sistema, $\beta$ o coeficiente de amortecimento estrutural (determinado experimentalmente) e $X$ a amplitude da resposta do sistema em regime permanente. Igualando-se as equações (3-12) e (3-13), obtêm-se o coeficiente de amortecimento linear viscoso equivalente para um sistema com amortecimento estrutural, dado por $c=\frac{k \beta}{\omega}$.

De acordo com [2], usando a representação exponencial complexa para um forçamento harmônico externo aplicado a um sistema com amortecimento 
linear viscoso, que é equivalente a um sistema com amortecimento estrutural, tem-se que a equação da dinâmica é dada por

$$
m \ddot{X}(t)+\frac{\beta k}{\omega} \dot{X}(t)+k X(t)=F_{0} e^{j \omega t},
$$

de modo que a resposta em regime permanente será $X(t)=X_{0} e^{j \omega t}$. De acordo com [2], substituindo $\dot{X}(t)=j \omega X_{0} e^{j \omega t}$ na equação (3-14), obtêm-se

$$
m \ddot{X}(t)+k(1+j \beta) X(t)=F_{0} e^{j \omega t} .
$$

Em [1], o coeficiente de amortecimento estrutural é definido como $h=k \beta$, de modo que a equação (3-15) é escrita como

$$
m \ddot{X}(t)+(k+j h) X(t)=F_{0} e^{j \omega t},
$$

e além disso, em [1], o termo $(k+j h)$ é definido como a rigidez complexa do sistema, denotada por $k_{c}$, de forma que a equação (3-16) passa a ser escrita como

$$
m \ddot{X}(t)+k_{c} X(t)=F_{0} e^{j \omega t} .
$$

Ainda de acordo com [1], a FRF para um sistema com amortecimento estrutural é dada por

$$
H(\omega)=\frac{1}{k-\omega^{2} m+j h} .
$$




\section{Sistemas com Múltiplos Graus de Liberdade}

\section{1}

\section{Sistemas sem Amortecimento}

Os sistemas mecânicos com múltiplos graus de liberdade necessitam de um tratamento vetorial, devido as várias coordenadas que representam cada um dos seus graus de liberdade. Tais sistemas, para um melhor entendimento da teoria, podem ser separados entre aqueles que possuem ou não amortecimento. Os sistemas mecânicos com múltiplos graus de liberdade com amortecimento ainda podem ser divididos em dois tipos: os que possuem amortecimento proporcional e os que possuem amortecimento não proporcional.

Os sistemas mecânicos com múltiplos graus de liberdade terão, assim como no caso dos sistemas com um grau de liberdade, a sua dinâmica governada por um P.V.I. Porém, neste caso, serão necessárias $n$ e.d.o's para descrever a sua dinâmica, cada uma referente a um grau de liberdade do sistema e, para cada uma dessas e.d.o's, serão necessárias duas C.I's.

Estas $n$ e.d.o's podem ser agrupadas em uma forma matricial, de modo a facilitar o seu estudo. Portanto, ao se reescrever as $n$ e.d.o's para um sistema mecânico com múltiplos graus de liberdade sem amortecimento, considerandose que este foi modelado como linear e invariante no tempo, obtêm-se a seguinte equação geral na forma matricial

$$
\mathbf{M} \ddot{\mathbf{X}}(t)+\mathbf{K X}(t)=\mathbf{U}(t)
$$

onde $\mathbf{X}(t) \in \mathbb{R}^{n \times 1}$ é um vetor de dimensão $n \times 1$, denominado vetor de deslocamentos, cujas componentes $x_{i}(t), i=1, \ldots, n$ representam os $n$ graus de liberdade do sistema. $\mathbf{M}$ e $\mathbf{K} \in \mathbb{R}^{n \times n}$ são matrizes constantes de dimensão $n \times n$, denominadas, respectivamente, matriz de massa e matriz de rigidez $\mathrm{e}$ $\mathbf{U}(t) \in \mathbb{R}^{n \times 1}$ é um vetor de dimensão $n \times 1$, denominado vetor de forçamentos, cuja componente $u_{i}(t), i=1, \ldots, n$, representa o forçamento $u_{i}(t)$ que age na massa $m_{i}$ do sistema.

Os sistemas mecânicos com múltiplos graus de liberdade apresentam um conceito importante conhecido como modo de vibração, que existe tanto para sistemas com amortecimento como para sistemas sem amortecimento. A obtenção dos modos de vibração é feita através do estudo da resposta livre do 
sistema. No caso de sistemas sem amortecimento, a resposta livre do sistema será a solução da equação homogênea associada a equação (4-1), que é dada por

$$
\mathbf{M} \ddot{\mathbf{X}}_{H}(t)+\mathbf{K X}_{H}(t)=\mathbf{0}
$$

cuja solução é conhecida e tem a forma $\mathbf{X}_{H}(t)=e^{j \omega t} \boldsymbol{\psi}$, onde $\boldsymbol{\psi} \in \mathbb{R}^{n \times 1}$ é um vetor de constantes de dimensão $n \times 1$.

Substituindo $\mathbf{X}_{H}(t)=e^{j \omega t} \boldsymbol{\psi}$ e sua segunda derivada na equação (4-2), e rearranjando os termos, obtêm-se

$$
\left(\mathbf{K}-\omega^{2} \mathbf{M}\right) \boldsymbol{\psi}=\mathbf{0}
$$

A equação (4-3) representa um problema de autovalor, cuja solução são as raízes do polinômio característico do sistema, obtidas através da equação $\operatorname{det}\left(\mathbf{K}-\omega^{2} \mathbf{M}\right)=0$. Portanto, as raízes do polinômio característico serão os autovalores da matriz $\mathbf{K}-\omega^{2} \mathbf{M}$ e como esta apresenta dimensão $n \times n$, existirão $n$ autovalores e consequentemente $n$ autovetores associados.

O autovalor $\omega_{i}^{2}, i=1, \ldots, n$ representa o quadrado da i-ésima frequência natural $\omega_{i}$ do sistema e o respectivo autovetor $\boldsymbol{\psi}_{i}$ representa o i-ésimo modo de vibração do sistema. Desta forma, um sistema com $n$ graus de liberdade apresentará $n$ frequências naturais e $n$ modos de vibração. Estes são definidos em [1] como modos principais, modos normais ou modos não amortecidos. De acordo com [1, se as $n$ frequências naturais forem não nulas e distintas, os modos de vibração serão linearmente independentes, e formarão uma base para o espaço vetorial de dimensão $n$.

As equações da dinâmica quando estão escritas no formato da equação (4-1) encontram-se acopladas, ou seja, elas apresentam mais de uma função incógnita, o que dificulta encontrar a solução do problema. Uma maneira de resolver isto, de acordo com [1], [2] e [3] é desacoplar as equações da dinâmica, de forma que cada uma delas apresente apenas uma função incógnita, facilitando a obtenção da solução analítica e da sua aproximação numérica.

O desacoplamento das equações da dinâmica será mostrado em resumo, baseando-se no que é feito em [1] e [3]. De acordo com a dedução apresentada por estes, pode-se chegar as seguintes relações para as matrizes de massa e rigidez partindo-se da equação (4-3)

$$
\boldsymbol{\psi}_{r}^{T} \mathbf{M} \boldsymbol{\psi}_{s}=0 \quad \forall r \neq s
$$




$$
\boldsymbol{\psi}_{r}^{T} \mathbf{K} \psi_{s}=0 \quad \forall r \neq s
$$

Como apontado em [3], estas equações afirmam que os modos de vibração são ortogonais em relação as matrizes de massa e de rigidez. Além destas relações, em [1], também obtêm-se que

$$
\begin{gathered}
\boldsymbol{\psi}_{r}^{T} \mathbf{M} \boldsymbol{\psi}_{r}=m_{r}, \\
\boldsymbol{\psi}_{r}^{T} \mathbf{K} \boldsymbol{\psi}_{r}=k_{r}, \\
\omega_{r}^{2}=\frac{k_{r}}{m_{r}},
\end{gathered}
$$

para $r=1, \ldots, n$, onde $m_{r}$ e $k_{r}$ são definidos, respectivamente, como massa modal e rigidez modal do r-ésimo modo, mas conforme afirmado em [1], estas grandezas não tem unidades de massa nem de rigidez.

Agrupando-se as frequências naturais e os modos de vibração em matrizes, como é apresentado em [1], [2] e [3], obtêm-se

$$
\begin{aligned}
& \boldsymbol{\Omega}^{2}=\left[\begin{array}{lll}
\omega_{1}^{2} & & \\
& \ddots & \\
& & \omega_{n}^{2}
\end{array}\right] \\
& \boldsymbol{\Psi}=\left[\begin{array}{lll}
\boldsymbol{\psi}_{1} & \ldots & \boldsymbol{\psi}_{n}
\end{array}\right]
\end{aligned}
$$

de forma que, como apresentado em [1] e [3], chega-se a duas novas matrizes

$$
\begin{gathered}
\boldsymbol{\Psi}^{T} \mathbf{M} \boldsymbol{\Psi}=\mathbf{M}_{m}, \\
\boldsymbol{\Psi}^{T} \mathbf{K} \boldsymbol{\Psi}=\mathbf{K}_{m},
\end{gathered}
$$

definidas, respectivamente, como matriz de massa modal e matriz de rigidez modal. Estas matrizes são diagonais, e os seus elementos são, respectivamente, as massas modais e as rigidezes modais. Além destas novas matrizes obtidas, também vale a seguinte relação apresentada em [1] e [3]

$$
\Omega^{2}=\mathbf{K}_{m} \mathbf{M}_{m}^{-1} .
$$


A próxima etapa para desacoplar as equações da dinâmica é escrever os modos normais em uma representação que seja única, pois como estes são dados pelos autovetores do problema de autovalor da equação (4-3), qualquer múltiplo de um desses autovetores também poderá ser considerado como um modo normal de vibração. Desta forma, em [1], define-se os modos massanormalizados como

$$
\phi_{r}=\frac{1}{\sqrt{m_{r}}} \boldsymbol{\psi}_{r} \quad r=1, \ldots, n
$$

dados pelos modos normais originais multiplicados pelo inverso da raiz quadrada das massas modais. Os modos massa-normalizados, quando agrupados em forma matricial, como também é feito em [1] e [3], são expressos como

$$
\boldsymbol{\Phi}=\boldsymbol{\Psi} \mathbf{M}_{m}^{-1 / 2}
$$

Como mostrado em [3], fazendo uma mudança de variável $\mathbf{X}(t)=\mathbf{\Phi} \mathbf{Y}(t)$ e substituindo na equação (4-1) e, após isto, pré-multiplicando-a por $\boldsymbol{\Phi}^{T}$ tem-se que

$$
\boldsymbol{\Phi}^{T} \mathbf{M} \boldsymbol{\Phi} \ddot{\mathbf{Y}}(t)+\boldsymbol{\Phi}^{T} \mathbf{K} \boldsymbol{\Phi} \mathbf{Y}(t)=\boldsymbol{\Phi}^{T} \mathbf{U}(t)
$$

e, conforme mostrado em [1] e [3], $\boldsymbol{\Phi}^{T} \mathbf{M} \boldsymbol{\Phi}=\mathbf{I}$ e $\boldsymbol{\Phi}^{T} \mathbf{K} \boldsymbol{\Phi}=\boldsymbol{\Omega}^{2}$, de forma que a equação (4-16) se torna

$$
\ddot{\mathbf{Y}}(t)+\Omega^{2} \mathbf{Y}(t)=\boldsymbol{\Phi}^{T} \mathbf{U}(t)
$$

que representa as equações de $n$ sistemas mecânicos com um grau de liberdade independentes entre si. Cada uma das equações presente em (4-17) pode ser resolvida individualmente, cada uma com sua respectiva C.I e após se obter o vetor de deslocamento $\mathbf{Y}(t)$ (que de acordo com [3], suas componentes são as coordenadas modais), obtêm-se a resposta do sistema $\mathbf{X}(t)$.

Este procedimento de desacoplar as equações da dinâmica, permite com que a resposta do sistema no domínio do tempo seja calculada de uma forma mais simples e direta, baseando-se na dinâmica de sistemas com um grau de liberdade.

Para analisar a resposta do sistema no domínio da frequência, de forma 
semelhante a que é feita para sistemas com um grau de liberdade, deve-se conhecer a matriz de FRF do sistema. Ela pode ser obtida de forma analítica estudando-se a resposta do sistema sob um forçamento harmônico conhecido. De acordo com o que é apresentado em [1] e [3], fazendo-se $\mathbf{U}(t)=\mathbf{U} \operatorname{sen}(\omega t)$ na equação (4-1), onde $\mathbf{U} \in \mathbb{R}^{n \times 1}$ é um vetor de constantes, cujas componentes representam as amplitudes dos forçamentos harmônicos aplicados em cada massa do sistema, obtêm-se

$$
\mathbf{M} \ddot{\mathbf{X}}(t)+\mathbf{K X}(t)=\mathbf{U} \operatorname{sen}(\omega t)
$$

que de acordo com [1] e [3], a solução particular será $\mathbf{X}(t)=\mathbf{X}_{0} \operatorname{sen}(\omega t)$. Substituindo $\mathbf{X}(t)$ e sua segunda derivada na equação (4-18), obtêm-se

$$
\left(-\omega^{2} \mathbf{M}+\mathbf{K}\right) \mathbf{X}_{0}=\mathbf{U}
$$

onde o termo $\left(-\omega^{2} \mathbf{M}+\mathbf{K}\right)$ é definido como a matriz de rigidez dinâmica e denotada em [3] por $\mathbf{K}_{\text {din }}(\omega)$. Para encontrar o vetor de constantes $\mathbf{X}_{0}$, cujas componentes são as amplitudes das respostas em regime permanente, de cada um dos graus de liberdade, multiplica-se ambos os lados da equação (4-19) pela inversa da matriz de rigidez dinâmica, obtendo-se

$$
\mathbf{X}_{0}=\mathbf{K}_{\operatorname{din}}^{-1}(\omega) \mathbf{U}
$$

A matriz $\mathbf{K}_{\text {din }}^{-1}(\omega)$ é definida como a matriz de FRF do sistema e denotada por $\mathbf{H}(\omega)$, tendo-se portanto que $\mathbf{X}_{0}=\mathbf{H}(\omega) \mathbf{U}$. Na matriz de FRF, cada um dos elementos $h_{i j}(\omega)$ são funções da frequência $\omega$ do forçamento externo. De acordo com [1] e [3], estas FRF's que compõem $\mathbf{H}(\omega)$ podem ser interpretadas como sendo a razão entre a amplitude da resposta em regime permanente do grau de liberdade $i$ e a amplitude do forçamento externo agindo no grau de liberdade $j$, considerando-se que só exista este forçamento agindo no sistema. Além disto, de acordo com [1] e [3], pode-se obter a FRF da velocidade e da aceleração, respectivamente, por $\mathbf{H}_{v}(\omega)=j \omega \mathbf{H}(\omega)$ e $\mathbf{H}_{a}(\omega)=-\omega^{2} \mathbf{H}(\omega)$.

Uma forma mais útil de representar a matriz de FRF é por um processo denominado decomposição modal, que procura escrever $\mathbf{H}(\omega)$ em função dos parâmetros modais (frequências naturais e modos de vibração). De acordo com o que é colocado em [1] e [3], a decomposição modal tem como objetivo facilitar o cálculo de $\mathbf{H}(\omega)$, pois quando esta é expressa como a inversa da matriz de 
rigidez dinâmica, a inversão de tal matriz se torna muito custosa e demorada, pois deve ser feita para cada valor de frequência do forçamento externo. Além disto, a decomposição modal, segundo [1] e [3], permite ver a influência dos modos de vibração e frequências naturais na resposta do sistema.

O procedimento mostrado em [3], para se obter a decomposição modal de $\mathbf{H}(\omega)$, parte da sua definição, escrita como $\mathbf{K}-\omega^{2} \mathbf{M}=\mathbf{H}^{-1}(\omega)$. Pré multiplicando esta equação por $\boldsymbol{\Phi}^{T}$ e pós multiplicando por $\boldsymbol{\Phi}$, e usando o fato de que $\boldsymbol{\Phi}^{T} \mathbf{M} \boldsymbol{\Phi}=\mathbf{I}$ e $\boldsymbol{\Phi}^{T} \mathbf{K} \boldsymbol{\Phi}=\boldsymbol{\Omega}^{2}$, obtêm-se

$$
\boldsymbol{\Omega}^{2}-\omega^{2} \mathbf{I}=\boldsymbol{\Phi}^{T} \mathbf{H}^{-1}(\omega) \boldsymbol{\Phi}
$$

e, de acordo com [3], invertendo ambos os lados da equação (4-21), têm-se

$$
\mathbf{H}(\omega)=\boldsymbol{\Phi}\left(\mathbf{\Omega}^{2}-\omega^{2} \mathbf{I}\right)^{-1} \boldsymbol{\Phi}^{T},
$$

que representa a decomposição modal da matriz de FRF em função dos parâmetros modais. De acordo com [3], cada um dos elementos da matriz de FRF, dada pela equação (4-22), são dados por

$$
h_{i j}(\omega)=\frac{\phi_{i 1} \phi_{j 1}}{\omega_{1}^{2}-\omega^{2}}+\frac{\phi_{i 2} \phi_{j 2}}{\omega_{2}^{2}-\omega^{2}}+\cdots+\frac{\phi_{i n} \phi_{j n}}{\omega_{n}^{2}-\omega^{2}},
$$

onde o termo $\phi_{i j}$ é o i-ésimo elemento do j-ésimo modo $\phi$ que compõem a matriz de modos massa-normalizados $\boldsymbol{\Phi}$.

\section{2}

\section{Sistemas com Amortecimento}

\subsection{1}

\section{Sistemas com Amortecimento Linear Viscoso}

Considerando agora os sistemas com amortecimento, estes podem ser divididos em dois tipos: proporcional e não proporcional. O amortecimento proporcional é aquele em que a matriz de amortecimento pode ser escrita como uma combinação linear (C.L) das matrizes de massa e rigidez.

Considerando o caso de amortecimento linear viscoso, as equações da dinâmica podem ser escritas na forma matricial por

$$
\mathbf{M} \ddot{\mathbf{X}}(t)+\mathbf{C X}(t)+\mathbf{K X}(t)=\mathbf{U}(t)
$$


e, se o amortecimento for proporcional, têm-se que $\mathbf{C}=\alpha \mathbf{M}+\beta \mathbf{K}$ é a matriz de amortecimento linear viscoso, $\operatorname{com} \alpha, \beta \in \mathbb{R}$. De acordo com [1], realizando o mesmo processo de desacoplamento do caso sem amortecimento, têm-se

$$
\boldsymbol{\Phi}^{T} \mathbf{M} \boldsymbol{\Phi} \ddot{\mathbf{Y}}(t)+\boldsymbol{\Phi}^{T} \mathbf{C} \boldsymbol{\Phi} \dot{\mathbf{Y}}(t)+\boldsymbol{\Phi}^{T} \mathbf{K} \boldsymbol{\Phi} \mathbf{Y}(t)=\boldsymbol{\Phi}^{T} \mathbf{U}(t)
$$

Como a matriz de amortecimento $\mathbf{C}$ é proporcional, obtêm-se que $\boldsymbol{\Phi}^{T} \mathbf{C} \boldsymbol{\Phi}=\boldsymbol{\Phi}^{T}(\alpha \mathbf{M}+\beta \mathbf{K}) \boldsymbol{\Phi}$. Desenvolvendo esta expressão, obtêm-se $\alpha \boldsymbol{\Phi}^{T} \mathbf{M} \boldsymbol{\Phi}+\beta \boldsymbol{\Phi}^{T} \mathbf{K} \boldsymbol{\Phi}=\alpha \mathbf{I}+\beta \boldsymbol{\Omega}^{2}$. Substituindo-a na equação (4-25), obtêm-se um conjunto de $n$ equações desacopladas dadas por

$$
\ddot{\mathbf{Y}}(t)+\left(\alpha \mathbf{I}+\beta \boldsymbol{\Omega}^{2}\right) \dot{\mathbf{Y}}(t)+\boldsymbol{\Omega}^{2} \mathbf{Y}(t)=\boldsymbol{\Phi}^{T} \mathbf{U}(t),
$$

que podem ser resolvidas individualmente, pois representam $n$ sistemas com um grau de liberdade independentes entre si. A r-ésima equação deste sistema de equações diferenciais pode ser escrita como

$$
\ddot{y}_{r}(t)+\left(\alpha+\beta \omega_{r}^{2}\right) \dot{y}_{r}(t)+\omega_{r}^{2} y_{r}(t)=\sum_{i=1}^{n} \phi_{i}^{r} U_{i}(t),
$$

onde $\phi_{i}^{r}$ é a i-ésima componente do r-ésimo modo $\phi$ (r-ésima coluna da matriz $\Phi)$. Como a equação (4-27) representa a dinâmica de um sistema com um grau de liberdade, de acordo com [1], a taxa de amortecimento será dada por $\zeta_{r}=\frac{\alpha}{2 \omega_{r}}+\frac{\beta \omega_{r}}{2}$. Além disso, de acordo com [1], os modos de vibração (modos normais) do sistema com amortecimento proporcional viscoso são os mesmos do caso sem amortecimento (para as mesmas matrizes de massa e rigidez).

No caso dos sistemas em que a matriz de amortecimento não pode ser escrita como uma C.L das matrizes de massa e rigidez (amortecimento não proporcional), de acordo com [1], as equações da dinâmica não podem ser desacopladas. Neste caso, para se obter a resposta do sistema do domínio do tempo, é preciso utilizar métodos numéricos para se calcular uma aproximação numérica para a solução do sistema de equações diferenciais (4-24). Neste caso, de acordo com [1], os modos de vibração e a frequências naturais do sistema terão valores complexos. Para se obter estes parâmetros modais, no caso de amortecimento não proporcional viscoso, deve-se analisar a resposta livre do sistema, ou seja analisar a equação homogênea associada a equação (4-24), que 
é dada por

$$
\mathbf{M} \ddot{\mathbf{X}}_{H}(t)+\mathbf{C} \dot{\mathbf{X}}_{H}(t)+\mathbf{K} \mathbf{X}_{H}(t)=\mathbf{0}
$$

De acordo com [1], a solução da equação (4-28) é $\mathbf{X}_{H}(t)=\boldsymbol{\xi} e^{s t}$, onde $s$ é a variável da transformada de Laplace (T.L) e $\boldsymbol{\xi}$ é um vetor cujas componentes assumem valores complexos, e representam as amplitudes dos deslocamentos referentes aos graus de liberdade do sistema. Substituindo $\mathbf{X}_{H}(t)$ e suas derivadas na equação (4-28), obtêm-se

$$
\left(s^{2} \mathbf{M}+s \mathbf{C}+\mathbf{K}\right) \boldsymbol{\xi}=\mathbf{0},
$$

que de acordo com [1], é um problema de autovalor complexo de ordem alta e deve ser resolvido utilizando-se a formulação em espaço de estados do sistema. Esta formulação cria um novo vetor de deslocamentos

$$
\mathbf{Y}(t)=\left[\begin{array}{c}
x(t) \\
\dot{x}(t)
\end{array}\right]
$$

de dimensões $2 n \times 1$, que agrupa todos os deslocamentos $x_{i}(t)$ e velocidades $\dot{x}_{i}(t), i=1, \ldots, n$ referentes aos graus de liberdade do sistema. De acordo com [1], a equação (4-28), quando escrita na formulação de espaço de estados, se torna

$$
[\mathbf{C}: \mathbf{M}] \dot{\mathbf{Y}}(t)+[\mathbf{K}: \mathbf{0}] \mathbf{Y}(t)=\mathbf{0},
$$

e usando a identidade $[\mathbf{M}: \mathbf{0}] \dot{\mathbf{Y}}(t)+[\mathbf{0}:-\mathbf{M}] \mathbf{Y}(t)=\mathbf{0}$, obtêm-se

$$
\left[\begin{array}{cc}
\mathbf{C} & \mathbf{M} \\
\mathbf{M} & \mathbf{0}
\end{array}\right] \dot{\mathbf{Y}}(t)+\left[\begin{array}{cc}
\mathbf{K} & \mathbf{0} \\
\mathbf{0} & -\mathbf{M}
\end{array}\right] \mathbf{Y}(t)=\mathbf{0}
$$

que pode ser escrita de forma simplificada como

$$
\mathbf{A} \dot{\mathbf{Y}}(t)+\mathbf{B Y}(t)=\mathbf{0}
$$


A equação (4-33), de acordo com [1], origina o seguinte problema de autovalor

$$
s_{r} \mathbf{A} \boldsymbol{\theta}_{r}+\mathbf{B} \boldsymbol{\theta}_{r}=\mathbf{0}
$$

cuja solução é dada por $2 n$ autovalores complexos $s_{r}$ e $2 n$ autovetores complexos $\boldsymbol{\theta}_{r}$ associados, ambos dados em pares de complexos conjugados.

Para se chegar na expressão da matriz de FRF do caso de amortecimento não proporcional viscoso, será apresentado o desenvolvimento realizado em [5].

Os autovalores $s_{r}$ e seus respectivos autovetores associados $\boldsymbol{\xi}_{r}$, satisfazem o problema de autovalor dado na equação (4-29) da seguinte forma

$$
\left(s_{r}^{2} \mathbf{M}+s_{r} \mathbf{C}+\mathbf{K}\right) \boldsymbol{\xi}_{r}=\mathbf{0},
$$

para $r=1, \ldots, 2 n$. De acordo com [5], a relação entre os autovetores $\boldsymbol{\theta}_{r}$ do sistema de primeira ordem (4-33) e os autovetores $\boldsymbol{\xi}_{r}$ do sistema de segunda ordem (4-28) é dada por

$$
\boldsymbol{\theta}_{r}=\left[\begin{array}{c}
\boldsymbol{\xi}_{r} \\
s_{r} \boldsymbol{\xi}_{r}
\end{array}\right] .
$$

Vale ressaltar que os autovetores $\boldsymbol{\xi}_{r}$ tem dimensão $n \times 1$. Multiplicando-se a equação (4-34) por $\boldsymbol{\theta}_{r}^{T}$, obtêm-se

$$
\boldsymbol{\theta}_{r}^{T} \mathbf{B} \boldsymbol{\theta}_{r}=-s_{r} \boldsymbol{\theta}_{r}^{T} \mathbf{A} \boldsymbol{\theta}_{r}
$$

onde, conforme definido em [5],

$$
\boldsymbol{\theta}_{r}^{T} \mathbf{A} \boldsymbol{\theta}_{r}=\frac{1}{\gamma_{r}}
$$

onde $\gamma_{r} \in \mathbb{C}$ é uma constante de normalização. Além disso, as propriedades de ortogonalidade dos autovetores do sistema de primeira ordem (4-33), de acordo com [5], são dadas por

$$
\begin{aligned}
\boldsymbol{\theta}_{j}^{T} \mathbf{A} \boldsymbol{\theta}_{k}=0 & \forall j \neq k, \\
\boldsymbol{\theta}_{j}^{T} \mathbf{B} \boldsymbol{\theta}_{k}=0 & \forall j \neq k .
\end{aligned}
$$


Usando a relação entre os autovetores dada na equação (4-36) e substituindo na equação (4-38), obtêm-se uma expressão para $\gamma_{r}$ em função dos autovetores $\boldsymbol{\xi}_{r}$ do sistema de segunda ordem (4-28), dada por

$$
\gamma_{r}=\frac{1}{\boldsymbol{\xi}_{r}^{T}\left[2 s_{r} \mathbf{M}+\mathbf{C}\right] \boldsymbol{\xi}_{r}} .
$$

Aplicando agora a formulação em espaço de estados na equação não homogênea (4-24), têm-se

$$
\mathbf{A} \dot{\mathbf{Y}}(t)+\mathbf{B Y}(t)=\mathbf{R}(t)
$$

onde $\mathbf{R}(t)=\left[\begin{array}{c}\mathbf{U}(t) \\ \mathbf{0}\end{array}\right]$. Aplicando a T.L em ambos os lados da equação (4-42), considerando C.I's não nulas, obtêm-se

$$
s \mathbf{A} \overline{\mathbf{Y}}(s)-\mathbf{A} \mathbf{Y}_{0}+\mathbf{B} \overline{\mathbf{Y}}(s)=\overline{\mathbf{R}}(s),
$$

onde $\overline{\mathbf{Y}}(s)=\left[\begin{array}{c}\overline{\mathbf{X}}(s) \\ s \overline{\mathbf{X}}(s)\end{array}\right]$ e $\overline{\mathbf{R}}(s)=\left[\begin{array}{c}\overline{\mathbf{U}}(s) \\ \mathbf{0}\end{array}\right]$ são, respectivamente, a T.L de $\mathbf{Y}(t)$ e $\mathbf{R}(t)$ e $\mathbf{Y}_{0}$ é um vetor de C.I's.

De acordo com [5], no caso em que os autovalores $s_{r}$ são distintos, a solução da equação (4-43) é dada por uma C.L dos modos $\boldsymbol{\theta}_{r}$. Desta forma, têm-se que

$$
\overline{\mathbf{Y}}(s)=\sum_{r=1}^{2 n} \beta_{r}(s) \boldsymbol{\theta}_{r}
$$

onde $\beta_{r}(s)$ são constantes a serem determinadas. Substituindo a equação (4-44) na equação (4-43) e rearranjando os termos, obtêm-se

$$
[s \mathbf{A}+\mathbf{B}] \sum_{r=1}^{2 n} \beta_{r}(s) \boldsymbol{\theta}_{r}=\overline{\mathbf{R}}(s)+\mathbf{A} \mathbf{Y}_{0}
$$

Multiplicando-se ambos os lados da equação (4-45) por $\boldsymbol{\theta}_{r}^{T}$, expandindo o somatório do lado esquerdo e aplicando-se as condições de ortogonalidade (4-39) e (4-40), obtêm-se os valores das constantes $\beta_{r}(s)$, dados por

$$
\beta_{r}(s)=\gamma_{r} \frac{\boldsymbol{\theta}_{r}^{T} \overline{\mathbf{R}}(s)+\boldsymbol{\theta}_{r}^{T} \mathbf{A} \mathbf{Y}_{0}}{\left(s-s_{r}\right)},
$$


com $r=1, \ldots, 2 n$. Substituindo a equação (4-36) no numerador da equação (4-46), obtêm-se uma expressão para as constantes $\beta_{r}(s)$ em função dos autovetores $\boldsymbol{\xi}_{r}$, dada por

$$
\beta_{r}(s)=\gamma_{r} \frac{\boldsymbol{\xi}_{r}^{T}\left(\overline{\mathbf{U}}(s)+\mathbf{M} \dot{\mathbf{X}}_{0}+\mathbf{C X}_{0}+s \mathbf{M} \mathbf{X}_{0}\right)}{\left(s-s_{r}\right)} .
$$

Desta forma, a partir da equação (4-44) e de (4-36), obtêm-se a resposta do sistema do domínio de Laplace, como sendo

$$
\overline{\mathbf{X}}(s)=\sum_{r=1}^{2 n} \beta_{r}(s) \boldsymbol{\xi}_{r},
$$

que, quando se substitui os valores das constantes $\beta_{r}(s)$ da equação (4-47), têm-se

$$
\overline{\mathbf{X}}(s)=\sum_{r=1}^{2 n} \gamma_{r} \boldsymbol{\xi}_{r} \frac{\boldsymbol{\xi}_{r}^{T}\left(\overline{\mathbf{U}}(s)+\mathbf{M} \dot{\mathbf{X}}_{0}+\mathbf{C X}_{0}+s \mathbf{M} \mathbf{X}_{0}\right)}{\left(s-s_{r}\right)}
$$

A partir da equação (4-49), têm-se, de acordo com [5], que a matriz de FRF do sistema com amortecimento não proporcional viscoso é dada por

$$
\mathbf{H}(s)=\sum_{r=1}^{2 n} \frac{\gamma_{r} \boldsymbol{\xi}_{r} \boldsymbol{\xi}_{r}^{T}}{\left(s-s_{r}\right)},
$$

onde $s=j \omega$ e $j=\sqrt{-1}$ é a unidade imaginária. Uma expressão semelhante a esta também é mostrada em [6]. Como os autovalores e autovetores são dados em pares de complexos conjugados, a equação (4-50) também é expressa em [5] como

$$
\mathbf{H}(s)=\sum_{r=1}^{n}\left[\frac{\gamma_{r} \boldsymbol{\xi}_{r} \boldsymbol{\xi}_{r}^{T}}{\left(s-s_{r}\right)}+\frac{\gamma_{r} \boldsymbol{\xi}_{r}^{*} \boldsymbol{\xi}_{r}^{* T}}{\left(s-s_{r}^{*}\right)}\right]
$$

onde $s_{r}^{*}$ e $\boldsymbol{\xi}_{r}^{*}$ são, respectivamente, os complexos conjugados de $s_{r}$ e $\boldsymbol{\xi}_{r}$. Segundo [5], expressando a FRF em termos da frequência natural complexa $\lambda_{r}$, têm-se que $s_{r}=j \lambda_{r}$, e portanto 


$$
\mathbf{H}(s)=\sum_{r=1}^{n}\left[\frac{\gamma_{r} \boldsymbol{\xi}_{r} \boldsymbol{\xi}_{r}^{T}}{\left(s-j \lambda_{r}\right)}+\frac{\gamma_{r} \boldsymbol{\xi}_{r}^{*} \boldsymbol{\xi}_{r}^{* T}}{\left(s+j \lambda_{r}\right)}\right]
$$

$\operatorname{com} \gamma_{r}=\frac{1}{\boldsymbol{\xi}_{r}^{T}\left[2 j \lambda_{r} \mathbf{M}+\mathbf{C}\right] \boldsymbol{\xi}_{r}}$.

A partir da equação (4-52), um elemento genérico $h_{i j}(s)$ da matriz de FRF é dado por

$$
h_{i j}(s)=\left[\frac{\gamma_{1} \xi_{i 1} \xi_{j 1}}{\left(s-j \lambda_{1}\right)}+\frac{\gamma_{1} \xi_{i 1}^{*} \xi_{j 1}^{*}}{\left(s+j \lambda_{1}\right)}\right]+\cdots+\left[\frac{\gamma_{n} \xi_{i n} \xi_{j n}}{\left(s-j \lambda_{n}\right)}+\frac{\gamma_{n} \xi_{i n}^{*} \xi_{j n}^{*}}{\left(s+j \lambda_{n}\right)}\right]
$$

onde $\xi_{i j}$ é o i-ésimo elemento do j-ésimo autovetor $\boldsymbol{\xi}_{j}$.

Conforme mostrado em [5], quando o sistema não tem amortecimento, ou seja, para $\mathbf{C}=\mathbf{0}$, têm-se que $\lambda_{r}=\lambda_{r}^{*}=\omega_{r}$ e $\boldsymbol{\xi}_{r}=\boldsymbol{\xi}_{r}^{*}=\boldsymbol{\phi}_{r}$, de modo

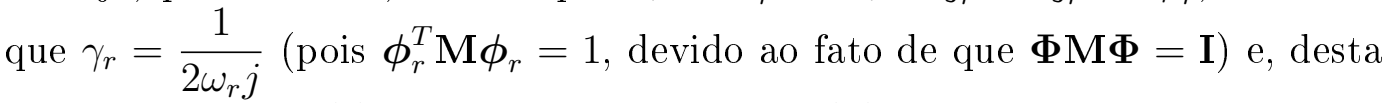
forma, a matriz $\mathbf{H}(s)$ se torna igual a matriz $\mathbf{H}(\omega)$ do caso sem amortecimento. Portanto, a equação (4-53) se reduz a equação (4-23) quando o amortecimento do sistema é nulo.

\subsection{2}

\section{Sistemas com Amortecimento Estrutural}

Considerando agora o amortecimento estrutural, este pode ser igualmente classificado em proporcional e não proporcional. De acordo com [1], a equação da dinâmica para o caso de amortecimento estrutural é dada por

$$
\mathbf{M} \ddot{\mathbf{X}}(t)+\mathbf{K X}(t)+j \mathbf{H X}(t)=\mathbf{0}
$$

onde $\mathbf{H}$ é a matriz de amortecimento estrutural. Se o amortecimento estrutural for proporcional, então $\mathbf{H}=\mu \mathbf{M}+\nu \mathbf{K}$, $\operatorname{com} \mu, \nu \in \mathbb{R}$. Segundo [1], neste caso, a matriz de modos será idêntica a do caso sem amortecimento e as frequências naturais serão dadas por

$$
\lambda_{r}^{2}=\omega_{r}^{2}\left(1+j \eta_{r}\right) \quad r=1, \ldots, n,
$$


onde $\eta_{r}=\nu+\frac{\mu}{\omega_{r}^{2}}$ é definido como sendo o fator de perda de amortecimento do r-ésimo modo.

Para o amortecimento estrutural não proporcional, a matriz $\mathbf{H}$ já não pode ser escrita como uma C.L de $\mathbf{M}$ e K. Neste caso, de acordo com [1], a solução da equação (4-54) é $\mathbf{X}(t)=\boldsymbol{\sigma} e^{j \lambda t}$, onde $\lambda$ é uma frequência complexa e $\boldsymbol{\sigma}$ um vetor de constantes complexas, cujas componentes representam as amplitudes dos deslocamentos referentes aos graus de liberdade do sistema. Substituindo $\mathbf{X}(t)$ e sua segunda derivada na equação (4-54), têm-se

$$
\left(\mathbf{K}_{c}-\lambda^{2} \mathbf{M}\right) \boldsymbol{\sigma}=\mathbf{0}
$$

onde $\mathbf{K}_{c}=\mathbf{K}+j \mathbf{H}$ é a matriz de rigidez complexa. De acordo com [1], a equação (4-56) representa um problema de autovalor complexo e a sua solução dará origem a uma matriz diagonal $\Lambda^{2}$ de autovalores e a uma matriz $\boldsymbol{\Sigma}$ de autovetores, ambas com valores complexos. Ainda segundo [1], as propriedades de ortogonalidade dos modos são dadas pelas equações

$$
\begin{aligned}
& \boldsymbol{\Sigma}^{T} \mathbf{M} \boldsymbol{\Sigma}=\mathbf{M}_{m}, \\
& \boldsymbol{\Sigma}^{T} \mathbf{K}_{c} \boldsymbol{\Sigma}=\mathbf{K}_{m}, \\
& \mathbf{K}_{m} \mathbf{M}_{m}=\mathbf{\Lambda}^{\mathbf{2}},
\end{aligned}
$$

onde a massa modal e a rigidez modal são complexas. Conforme é apresentado em [1], pode-se definir modos massa-normalizados a partir da matriz de massa modal para um sistema cujo o amortecimento é modelado como sendo estrutural não proporcional. Desta forma, para um sistema com tais características, a matriz de modos massa-normalizados é definida como sendo

$$
\Phi=\mathbf{M}_{m}^{-1 / 2} \boldsymbol{\Sigma}
$$

onde $\boldsymbol{\Phi}$ apresenta componentes com valores complexos.

De acordo com [1], a matriz de FRF para sistemas com amortecimento pode ser definida da mesma forma que para sistemas sem amortecimento, ou seja, como sendo a matriz inversa da matriz de rigidez dinâmica. Considerando o caso de amortecimento estrutural não proporcional, a matriz de FRF é dada por 


$$
\mathbf{H}(\omega)=\boldsymbol{\Phi}\left(\Lambda^{2}-\omega^{2} \mathbf{I}\right)^{-1} \boldsymbol{\Phi}^{T},
$$

que de acordo com [1], um elemento $h_{i j}(\omega)$ de $\mathbf{H}(\omega)$ é dado por

$$
h_{i j}(\omega)=\frac{\phi_{i 1} \phi_{j 1}}{\lambda_{1}^{2}-\omega^{2}}+\frac{\phi_{i 2} \phi_{j 2}}{\lambda_{2}^{2}-\omega^{2}}+\cdots+\frac{\phi_{i n} \phi_{j n}}{\lambda_{n}^{2}-\omega^{2}},
$$

sendo que $\lambda_{i}$ e $\phi_{i j}$, o i-ésimo elemento do j-ésimo modo $\phi$, apresentam valores complexos. 


\section{Modelagem Analítica}

5.1

Sistema 1

O primeiro sistema mecânico tratado é mostrado na figura 5.1

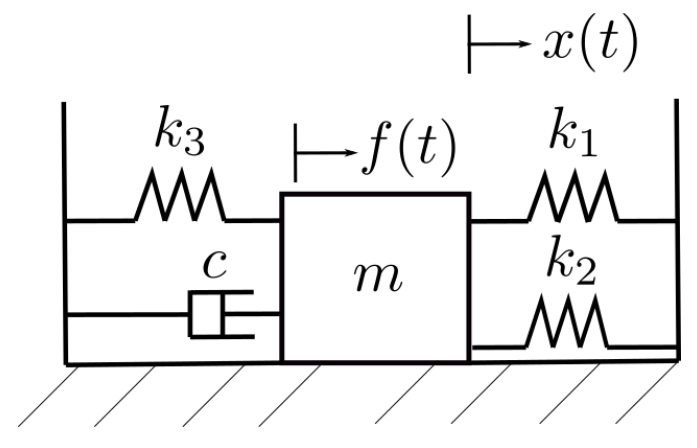

Figura 5.1: sistema 1.

onde a massa $m$ move-se sobre uma superfície sem atrito, $x(t)$ é medido a partir da posição em que a mola está relaxada e $f(t)=F_{0} \operatorname{sen}(\omega t)$ é o forçamento externo aplicado ao sistema.

Para obter a equação da dinâmica, deve-se primeiramente escrever o Lagrangeano do sistema que, neste caso, é dado por

$$
L=\frac{1}{2} m \dot{x}^{2}-\frac{1}{2} k x^{2}
$$

onde $k=k_{1}+k_{2}+k_{3}$. Desta forma, obtêm-se

$$
\begin{gathered}
\frac{d}{d t}\left(\frac{\partial L}{\partial \dot{x}}\right)=m \ddot{x}, \\
\frac{\partial L}{\partial x}=-k x, \\
Q=F_{0} \operatorname{sen}(\omega t), \\
D=\frac{1}{2} c \dot{x}^{2} \\
\frac{\partial D}{\partial \dot{x}}=c \dot{x}
\end{gathered}
$$


de modo que a equação obtida para a dinâmica do sistema é

$$
m \ddot{x}+c \dot{x}+k x=F_{0} \operatorname{sen}(\omega t) .
$$

Neste caso, como o forçamento externo é dado por uma função trigonométrica, a integral da convolução se torna trabalhosa, de modo que a resposta do sistema no domínio do tempo foi obtida analíticamente aplicando-se o método dos coeficientes indeterminados. De acordo com a teoria de equações diferenciais, sabe-se que a solução da equação homogênea associada a equação (5-7) é $x_{H}(t)=e^{\lambda t}$, onde $\lambda$ é um valor constante. Substituindo $x_{H}(t)$ e suas derivadas na equação homogênea e resolvendo para $\lambda$, obtêm-se

$$
\lambda=\frac{-c \pm \sqrt{c^{2}-4 m k}}{2 m} .
$$

Os parâmetros do sistema foram definidos de modo que se tivesse $\Delta=c^{2}-4 m k=0$ que, de acordo com a teoria de vibrações, representa o caso de vibração críticamente amortecida, que equivale também a se ter a taxa de amortecimento $\zeta=1$. Desta forma, tem-se que $\lambda=\frac{-c}{2 m}$. Neste caso, sabe-se pela teoria de equações diferenciais que $x_{H}(t)=A e^{\lambda t}+B t e^{\lambda t}$, onde $A$ e $B$ são constantes a serem determinadas utilizando-se as C.I's.

A solução particular $x_{p}(t)$ da equação não homogênea, representa a resposta do sistema em regime permanente e, no caso de sistemas mecânicos lineares, considerando que não há ressonância, apresenta a mesma forma do forçamento externo. Portanto, $x_{p}(t)=C \operatorname{sen}(\omega t+\theta)$, onde $C$ e $\theta$ são constantes a serem determinadas. Neste caso, é necessário considerar uma diferença de fase $\theta$ entre a resposta do sistema em regime permanente e o forçamento externo pois, de acordo com a teoria de vibrações, quando o sistema apresenta amortecimento sempre existirá uma diferença de fase entre 0 e $\pi$ radianos entre a resposta em regime permanente e o forçamento externo.

A solução particular pode ser reescrita como

$$
x_{p}(t)=C(\operatorname{sen}(\omega t) \cos (\theta)+\operatorname{sen}(\theta) \cos (\omega t)) \text {, }
$$

que rearranjando os termos se torna

$$
x_{p}(t)=D \operatorname{sen}(\omega t)+E \cos (\omega t),
$$


onde $D=C \cos (\theta)$ e $E=C \operatorname{sen}(\theta)$ são constantes. Substituindo a equação (5-10) e suas derivadas na equação (5-7), obtêm-se

$$
\begin{gathered}
D=\frac{F_{0}}{\left(k-m \omega^{2}\right)-\frac{c^{2} \omega^{2}}{m \omega^{2}-k}}, \\
E=\frac{c \omega F_{0}}{\left(k-m \omega^{2}\right)\left(m \omega^{2}-k\right)-c^{2} \omega^{2}} .
\end{gathered}
$$

Desta forma, tem-se que $x(t)=A e^{\lambda t}+B t e^{\lambda t}+D \operatorname{sen}(\omega t)+E \cos (\omega t)$. Considerando C.I's nulas, ou seja, $x(0)=0$ e $\dot{x}(0)=0$, obtêm-se que $A=-E$ e $B=E \lambda-D \omega$.

Para encontrar a resposta do sistema no domínio da frequência, devese determinar a sua FRF, que no caso é dada pela equação (3-10). Portanto, utilizando-se a FRF, obtêm-se a amplitude da resposta em regime permanente, dada por $X_{0}=F_{0}|H(\omega)|$, onde $|H(\omega)|$ é o módulo da FRF, dado por

$$
|H(\omega)|=\sqrt{\operatorname{Re}(H(\omega))^{2}+\operatorname{Img}(H(\omega))^{2}},
$$

onde $\operatorname{Re}(H(\omega))$ e $\operatorname{Img}(H(\omega))$ são, respectivamente, a parte real e imaginária de $H(\omega)$, obtidas multiplicando-se o numerador e o denominador da FRF pelo conjugado do denominador. Fazendo isto, obtêm-se

$$
\begin{aligned}
\operatorname{Re}(H(\omega)) & =\frac{k-m \omega^{2}}{m^{2} \omega^{4}-2 m k \omega^{2}+c^{2} \omega^{2}+k^{2}}, \\
\operatorname{Img}(H(\omega)) & =\frac{-c \omega}{m^{2} \omega^{4}-2 m k \omega^{2}+c^{2} \omega^{2}+k^{2}} .
\end{aligned}
$$

Deste modo, para diferentes valores da frequência $\omega$ do forçamento externo, irá se obter diferentes amplitudes $X_{0}$ da resposta do sistema em regime permanente, mostrando como que o forçamento externo influencia no comportamento dinâmico do sistema. As partes real e imaginária também podem ser obtidas para a FRF no formato da equação (3-11), sendo dadas por 


$$
\begin{aligned}
\operatorname{Re}(H(\omega)) & =\frac{1-r^{2}}{k r^{2}\left(\frac{1}{r^{2}}-2+r^{2}+4 \zeta^{2}\right)}, \\
\operatorname{Img}(H(\omega)) & =\frac{-2 \zeta r}{k r^{2}\left(\frac{1}{r^{2}}-2+r^{2}+4 \zeta^{2}\right)} .
\end{aligned}
$$

\section{2}

\section{Sistema 2}

O segundo sistema mecânico analisado é mostrado na figura 5.2 e apresenta cinco graus de liberdade $x_{i}(t), i=1, \ldots, 5$ medidos a partir da posição em que as molas encontram-se relaxadas. O forçamento externo $f(t)=F_{0} \operatorname{sen}(\omega t)$ é aplicado na maior massa do sistema, cujo movimento é restrito por um trilho sem atrito, impedindo que esta rotacione. Além disso, na modelagem realizada, não foi considerado o efeito da força gravitacional agindo nas massas do sistema.

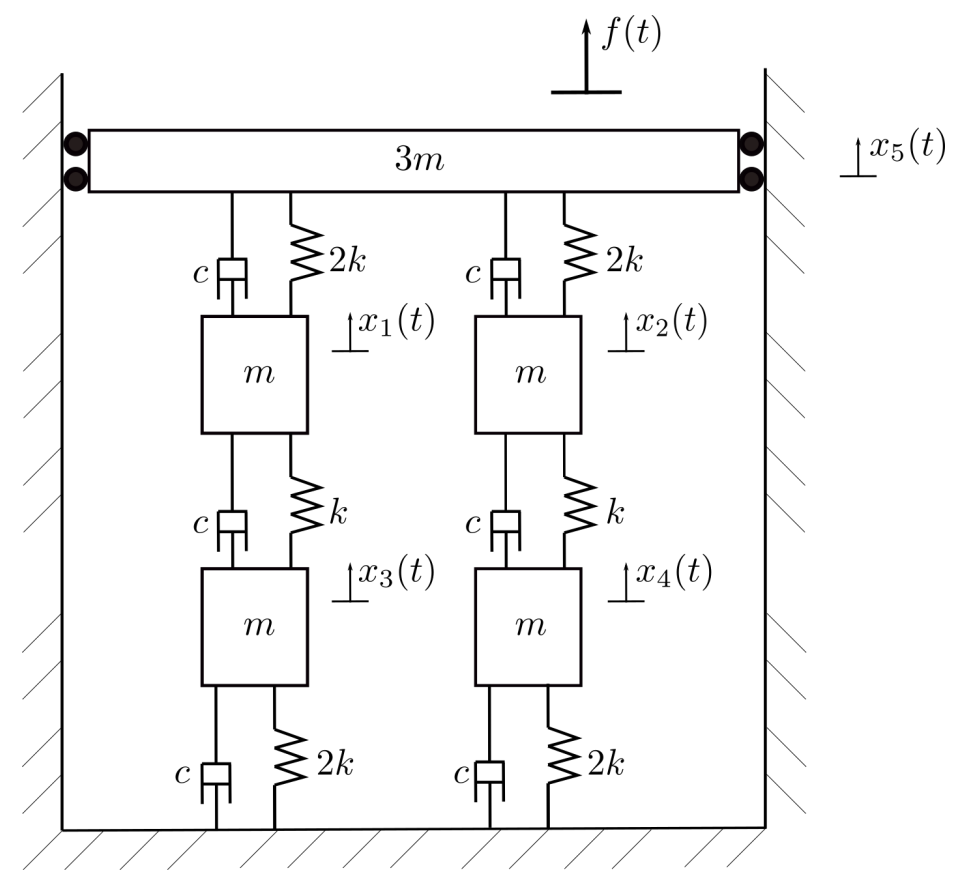

Figura 5.2: sistema 2.

O Lagrangeano obtido para o segundo sistema é dado por 


$$
\begin{aligned}
& L=\frac{1}{2} m\left(\dot{x}_{1}^{2}+\dot{x}_{2}^{2}+\dot{x}_{3}^{2}+\dot{x}_{4}^{2}+3 \dot{x}_{5}^{2}\right)- k\left[\left(x_{5}-x_{1}\right)^{2}+\left(x_{5}-x_{2}\right)^{2}+x_{3}^{2}+x_{4}^{2}\right] \\
&-\frac{1}{2} k\left[\left(x_{1}-x_{3}\right)^{2}+\left(x_{2}-x_{4}\right)^{2}\right], \quad(5-18
\end{aligned}
$$

de modo que, para $x_{1}(t)$

$$
\begin{gathered}
\frac{d}{d t}\left(\frac{\partial L}{\partial \dot{x}_{1}}\right)=m \ddot{x}_{1}, \\
\frac{\partial L}{\partial x_{1}}=2 k\left(x_{5}-x_{1}\right)-k\left(x_{1}-x_{3}\right), \\
Q_{1}=0, \\
D=\frac{1}{2} c\left(\dot{x}_{5}-\dot{x}_{1}\right)^{2}+\frac{1}{2} c\left(\dot{x}_{1}-\dot{x}_{3}\right)^{2}, \\
\frac{\partial D}{\partial \dot{x}_{1}}=-c\left(\dot{x}_{5}-\dot{x}_{1}\right)+c\left(\dot{x}_{1}-\dot{x}_{3}\right),
\end{gathered}
$$

para $x_{2}(t)$

$$
\begin{gathered}
\frac{d}{d t}\left(\frac{\partial L}{\partial \dot{x}_{2}}\right)=m \ddot{x}_{2}, \\
\frac{\partial L}{\partial x_{2}}=2 k\left(x_{5}-x_{2}\right)-k\left(x_{2}-x_{4}\right), \\
Q_{2}=0, \\
D=\frac{1}{2} c\left(\dot{x}_{5}-\dot{x}_{2}\right)^{2}+\frac{1}{2} c\left(\dot{x}_{2}-\dot{x}_{4}\right)^{2}, \\
\frac{\partial D}{\partial \dot{x}_{2}}=-c\left(\dot{x}_{5}-\dot{x}_{2}\right)+c\left(\dot{x}_{2}-\dot{x}_{4}\right),
\end{gathered}
$$

para $x_{3}(t)$

$$
\begin{gathered}
\frac{d}{d t}\left(\frac{\partial L}{\partial \dot{x}_{3}}\right)=m \ddot{x}_{3} \\
\frac{\partial L}{\partial x_{3}}=-2 k x_{3}+k\left(x_{1}-x_{3}\right), \\
Q_{3}=0 \\
D=\frac{1}{2} c \dot{x}_{3}^{2}+\frac{1}{2} c\left(\dot{x}_{1}-\dot{x}_{3}\right)^{2}, \\
\frac{\partial D}{\partial \dot{x}_{3}}=c \dot{x}_{3}-c\left(\dot{x}_{1}-\dot{x}_{3}\right),
\end{gathered}
$$


para $x_{4}(t)$

$$
\begin{gathered}
\frac{d}{d t}\left(\frac{\partial L}{\partial \dot{x}_{4}}\right)=m \ddot{x}_{4}, \\
\frac{\partial L}{\partial x_{4}}=-2 k x_{4}+k\left(x_{2}-x_{4}\right), \\
Q_{4}=0, \\
D=\frac{1}{2} c \dot{x}_{4}^{2}+\frac{1}{2} c\left(\dot{x}_{2}-\dot{x}_{4}\right)^{2}, \\
\frac{\partial D}{\partial \dot{x}_{4}}=c \dot{x}_{4}-c\left(\dot{x}_{2}-\dot{x}_{4}\right),
\end{gathered}
$$

e para $x_{5}(t)$

$$
\begin{gathered}
\frac{d}{d t}\left(\frac{\partial L}{\partial \dot{x}_{5}}\right)=3 m \ddot{x}_{5} \\
\frac{\partial L}{\partial x_{5}}=-2 k\left(x_{5}-x_{1}\right)-2 k\left(x_{5}-x_{2}\right), \\
Q_{5}=f(t) \\
D=\frac{1}{2} c\left(\dot{x}_{5}-\dot{x}_{1}\right)^{2}+\frac{1}{2} c\left(\dot{x}_{5}-\dot{x}_{2}\right)^{2} \\
\frac{\partial D}{\partial \dot{x}_{5}}=c\left(\dot{x}_{5}-\dot{x}_{1}\right)+c\left(\dot{x}_{5}-\dot{x}_{2}\right) .
\end{gathered}
$$

Após calcular os termos da equação (2-5), a partir do Lagrangeano, para cada um dos graus de liberdade, obteve-se as seguintes equações que descrevem a dinâmica do sistema

$$
\begin{gathered}
m \ddot{x}_{1}+2 c \dot{x}_{1}-c \dot{x}_{3}-c \dot{x}_{5}+3 k x_{1}-k x_{3}-2 k x_{5}=0, \\
m \ddot{x}_{2}+2 c \dot{x}_{2}-c \dot{x}_{4}-c \dot{x}_{5}+3 k x_{2}-k x_{4}-2 k x_{5}=0, \\
m \ddot{x}_{3}-c \dot{x}_{1}+2 c \dot{x}_{3}-k x_{1}+3 k x_{3}=0, \\
m \ddot{x}_{4}-c \dot{x}_{2}+2 c \dot{x}_{4}-k x_{2}+3 k x_{4}=0
\end{gathered}
$$

$$
3 m \ddot{x}_{5}-c \dot{x}_{1}-c \dot{x}_{2}+2 c \dot{x}_{5}-2 k x_{1}-2 k x_{2}+4 k x_{5}=f(t) .
$$


Escrevendo este conjunto de equações na forma matricial dada pela equação (4-24), obtêm-se que

$$
\begin{aligned}
& \mathbf{M}=\left[\begin{array}{ccccc}
m & 0 & 0 & 0 & 0 \\
0 & m & 0 & 0 & 0 \\
0 & 0 & m & 0 & 0 \\
0 & 0 & 0 & m & 0 \\
0 & 0 & 0 & 0 & 3 m
\end{array}\right], \quad \mathbf{C}=\left[\begin{array}{ccccc}
2 c & 0 & -c & 0 & -c \\
0 & 2 c & 0 & -c & -c \\
-c & 0 & 2 c & 0 & 0 \\
0 & -c & 0 & 2 c & 0 \\
-c & -c & 0 & 0 & 2 c
\end{array}\right], \\
& \mathbf{K}=\left[\begin{array}{ccccc}
3 k & 0 & -k & 0 & -2 k \\
0 & 3 k & 0 & -k & -2 k \\
-k & 0 & 3 k & 0 & 0 \\
0 & -k & 0 & 3 k & 0 \\
-2 k & -2 k & 0 & 0 & 4 k
\end{array}\right],
\end{aligned}
$$

com os vetores de deslocamento e forçamento dados por

$$
\mathbf{X}(t)=\left[\begin{array}{c}
x_{1}(t) \\
x_{2}(t) \\
x_{3}(t) \\
x_{4}(t) \\
x_{5}(t)
\end{array}\right], \quad \mathbf{U}(t)=\left[\begin{array}{c}
0 \\
0 \\
0 \\
0 \\
f(t)
\end{array}\right]
$$




\section{Simulação Numérica e Análise dos Resultados}

6.1

Sistema 1

O sistema 1 foi simulado no MatLab, obtendo-se a resposta no domínio do tempo e da frequência. Para obter uma aproximação numérica da solução do P.V.I dado pela equação (5-7) com C.I's nulas, teve-se que reescrever a equação (5-7) como um sistema de duas e.d.o's de primeira ordem, para que esta pudesse ser implementada no MatLab. Para isto, utilizou-se a formulação em espaço de estados do sistema, onde obteve-se a seguinte equação matricial

$$
\dot{\mathbf{Y}}(t)=\left[\begin{array}{cc}
0 & 1 \\
-\frac{k}{m} & -\frac{c}{m}
\end{array}\right] \mathbf{Y}(t)+\left[\begin{array}{c}
0 \\
\frac{F_{0}}{m} \operatorname{sen}(\omega t)
\end{array}\right],
$$

onde $\mathbf{Y}(t)=\left[\begin{array}{c}x(t) \\ \dot{x}(t)\end{array}\right]$ é o vetor de estados.

A equação (6-1) foi integrada numericamente no MatLab, utilizando-se o comando ode45, que implementa o método de Runge-Kutta de quarta ordem, para o qual se utilizou um passo de tempo $\Delta t=10^{-3} \mathrm{~s}$ e considerando $\mathbf{Y}_{0}(t)=\left[\begin{array}{l}0 \\ 0\end{array}\right]$ como o vetor de C.I's de posição e velocidade.

O resultado obtido é dado na figura 6.1, mostrando a solução analítica e a aproximação numérica da solução do P.V.I no domínio do tempo.

Os valores assumidos para os parâmetros do sistema foram: $m=2 \mathrm{~kg}$; $k_{1}=k_{3}=0.5 \mathrm{~N} / \mathrm{m} ; k_{2}=1.5 \mathrm{~N} / \mathrm{m}$ e $c=c_{c r i}=2 m \omega_{n}$, de modo que o sistema fosse críticamente amortecido. O forçamento externo foi assumido como sendo $f(t)=5 \operatorname{sen}(2 t) N$.

Observando o gráfico, conclui-se que a solução analítica e a aproximação numérica do P.V.I coincidem, conforme o esperado. A resposta no domínio da frequência é dada através dos gráficos que se originam a partir da FRF do sistema. O gráfico de Bode, os gráficos real e imaginário e o gráfico de Nyquist são dados, respectivamente, nas figuras 6.2 , 6.3 e 6.4 .

A amplitude da resposta em regime permanente foi calculada a partir da equação (5-13), e o resultado obtido é dado na figura 6.5. 


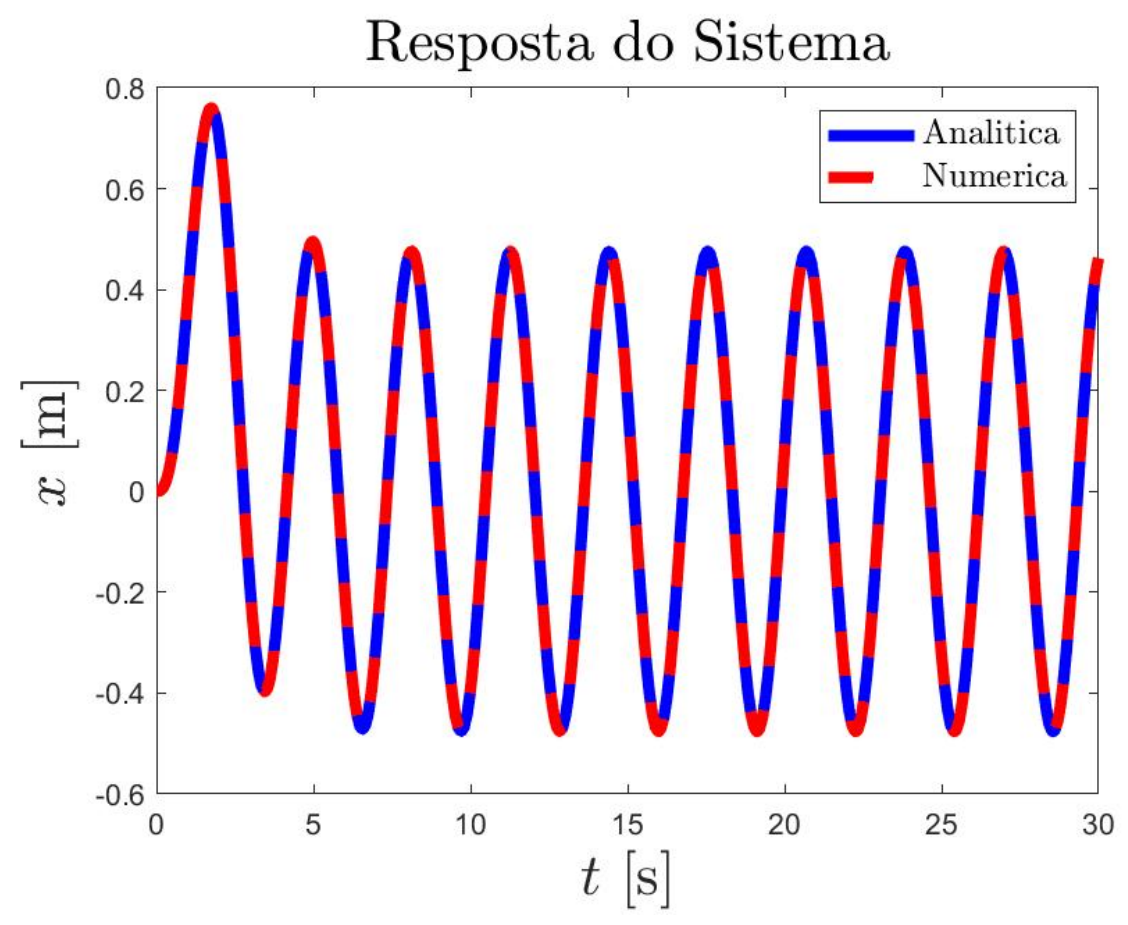

Figura 6.1: resposta no tempo do sistema 1.
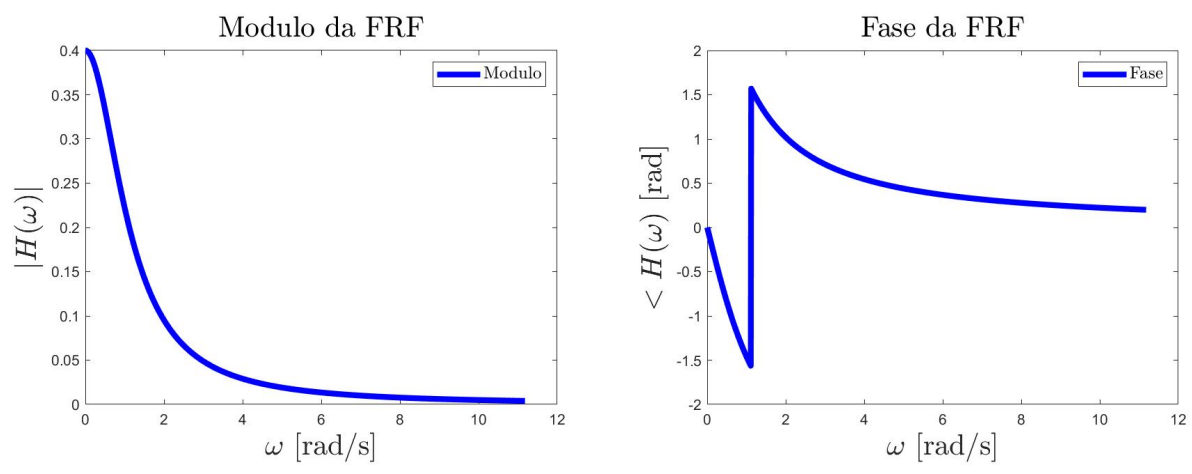

Figura 6.2: gráfico de Bode do sistema 1.
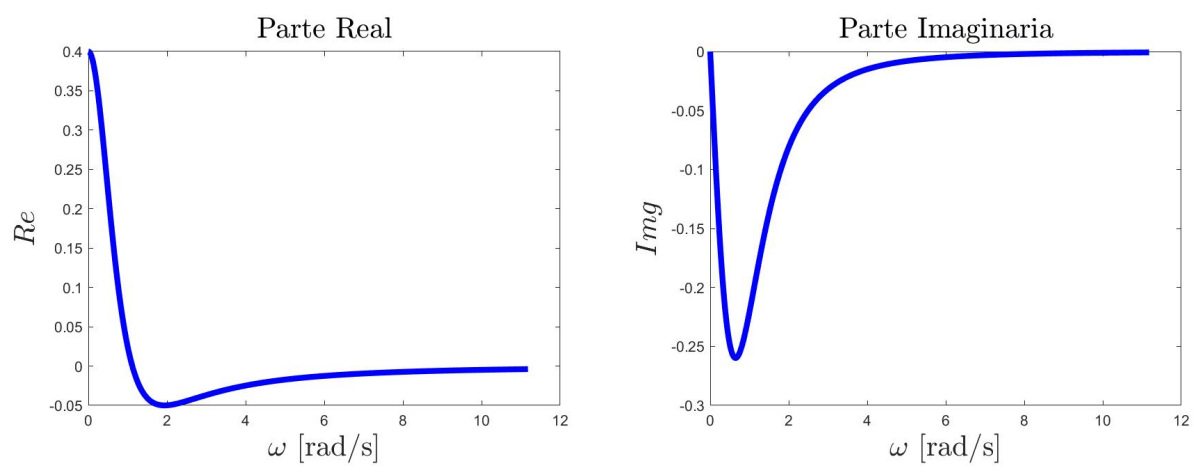

Figura 6.3: gráfico real e imaginário do sistema 1. 


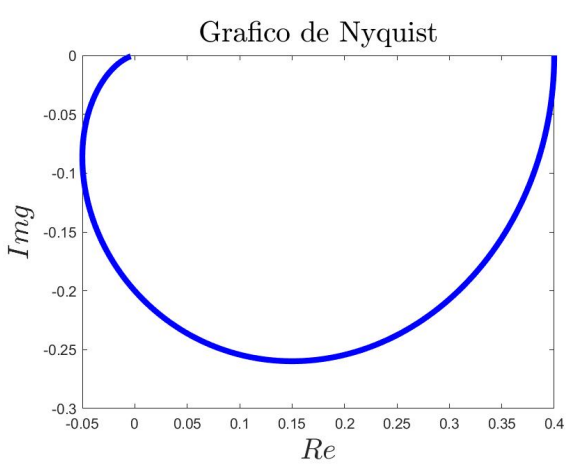

Figura 6.4: gráfico de Nyquist do sistema 1.

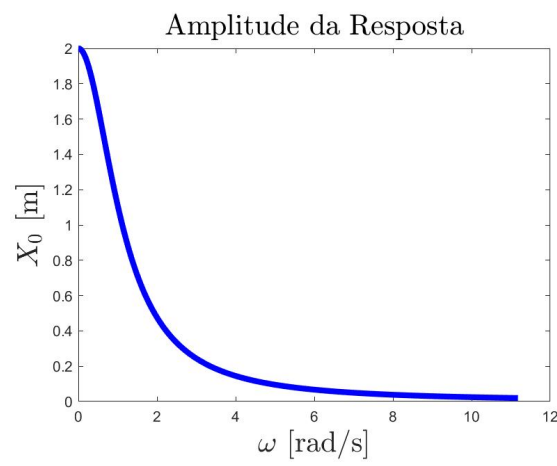

Figura 6.5: amplitude da resposta em regime permanente do sistema 1.

onde observa-se que este apresenta a mesma forma do gráfico de $|H(\omega)|$, como esperado, mudando apenas por um fator igual a amplitude $F_{0}=5$ do forçamento externo. Nota-se também que, para a frequência do forçamento externo $\omega=2 \mathrm{rad} / \mathrm{s}$, o valor de $X_{0}$ obtido corresponde ao valor da amplitude de $x(t)$ no gráfico mostrado na figura 6.1, pois este é o valor da amplitude da resposta do sistema quando este atinge o regime permanente. É interessante notar que, com o aumento da frequência do forçamento externo, a resposta do sistema em regime permanente assume valores cada vez menores, indicando que o sistema vai tendendo ao equilíbrio à medida em que se aplica forças em frequências mais elevadas.

Obteve-se também, a partir das equações (5-16) e (5-17), o gráfico que mostra a amplitude da resposta em regime permanente para diferentes valores da taxa de amortecimento $\zeta$, em função da razão de frequências $r$. O resultado é dado na figura 6.6 .

A curva em vermelho representa o sistema analisado, pois este é críticamente amortecido, ou seja $c=c_{c r i}=2 m \omega_{n}$, de forma que $\zeta=1$.

As curvas em azul, verde e amarelo, foram obtidas para $0<\zeta<1$, e representam o caso denominado vibração subamortecida, o qual $0<c<c_{c r i}$. 


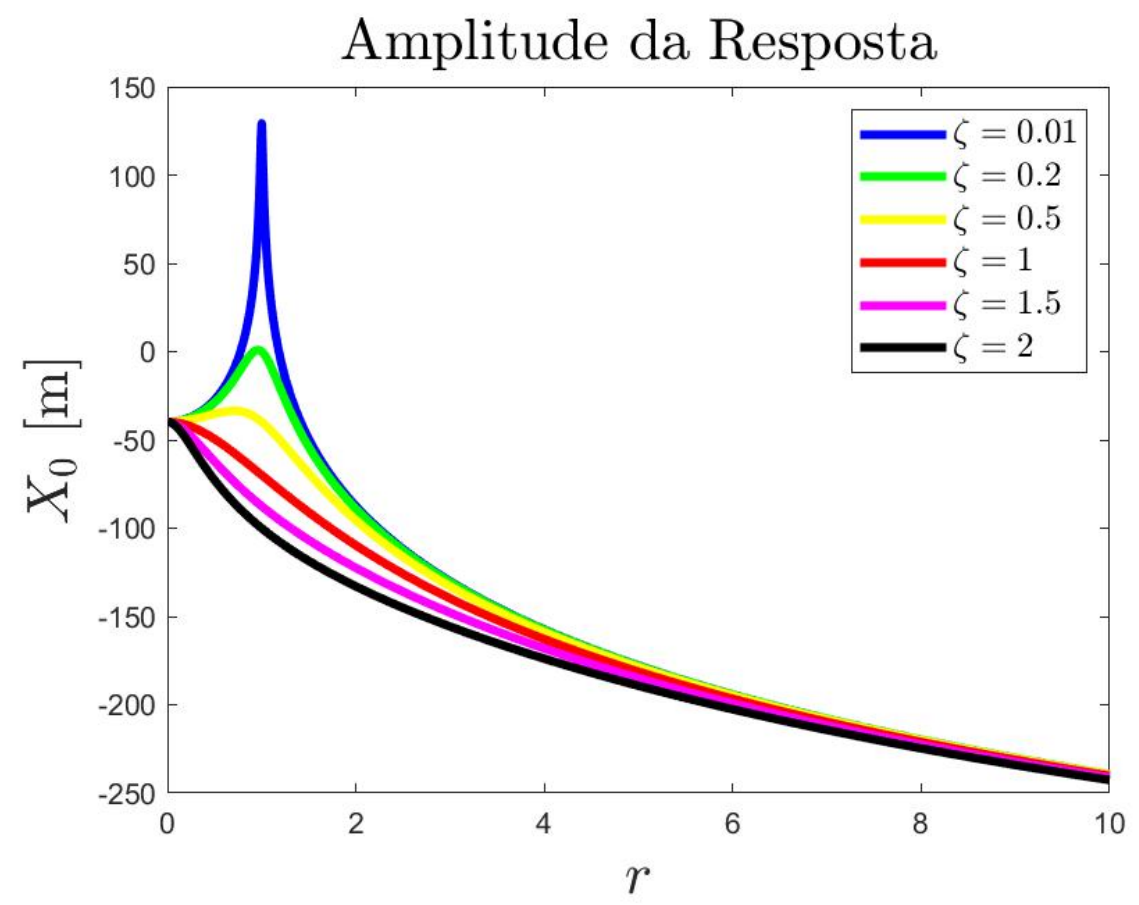

Figura 6.6: amplitude da resposta em regime permanente do sistema 1, para diferentes valores de $\zeta$.

As curvas rosa e preta, abaixo da curva de amortecimento crítico, foram obtidas para $\zeta>1$, e representam o caso denominado vibração superamortecida, o qual $c>c_{\text {cri }}$.

O caso não amortecido, ou seja, quando $c=0$, não pode ser obtido, pois quando $\zeta=0$ e $r=1$, a FRF dada pela equação (3-11) assume um valor infinito, o qual não pode ser representado graficamente, levando a amplitude da resposta em regime permanente ao mesmo comportamento. Este fenômeno é denominado ressonância, ou seja, quando tem-se um sistema com um grau de liberdade não amortecido, e este é excitado por uma força harmônica externa, cuja frequência $\omega_{f}$ é igual a frequência natural $\omega_{n}$ do sistema, este oscila em regime permanente com uma amplitude extremamente elevada.

Desta forma, a partir do gráfico da figura 6.6, observa-se que, à medida que a constante de amortecimento $c$ aumenta, as curvas apresentam uma diminuição no seu valor máximo, mostrando que quanto mais o sistema dissipa energia menores são as amplitudes da resposta em regime permanente, e que quanto mais próxima a frequência do forçamento externo estiver da frequência natural do sistema, maiores serão as amplitudes da resposta em regime permanente.

$\mathrm{Na}$ simulação das curvas da figura 6.6, teve-se que usar uma mudança de escala no eixo vertical, pois, de acordo com [1], quando há ressonância, estas 
impedem a vizualização da curva da FRF por completo, pois os demais valores são muito menores que os valores na ressonância. Porém, apesar do gráfico não mostrar o fenômeno da ressonância, ainda se faz necessário o uso de uma mudança de escala. Desta forma, baseando-se em [1], o valores da amplitude mostrados na figura 6.6 foram calculados como sendo $X_{0}=F_{0} \cdot 20 \log (|H(\omega)|)$ onde, segundo [1], $20 \log (|H(\omega)|)$ é o valor de $|H(\omega)|$ em escala decibel. Portanto, o real valor da amplitude é

$$
X_{0}=F_{0} \cdot 10^{\left(\frac{X_{g r}}{20 F_{0}}\right)},
$$

onde $X_{g r}$ é o valor dado no gráfico da figura 6.6.

\section{2}

\section{Sistema 2 sem Amortecimento}

O sistema 2 também foi simulado no MatLab, onde obteve-se as respostas no domínio do tempo e da frequência. Primeiro foi considerado o caso em que o amortecimento é nulo. Desta forma, após ter calculado a matriz de frequências naturais $\Omega^{2}$ e a matriz de modos massa-normalizados $\Phi$ com o auxílio do MatLab, obteve-se as seguintes frequências naturais para o sistema 2

$$
\begin{aligned}
& \omega_{1} \approx 0.584 \mathrm{rad} / \mathrm{s}, \\
& \omega_{2} \approx 1.732 \mathrm{rad} / \mathrm{s}, \\
& \omega_{3} \approx 1.975 \mathrm{rad} / \mathrm{s}, \\
& \omega_{4} \approx 2.449 \mathrm{rad} / \mathrm{s}, \\
& \omega_{5} \approx 2.599 \mathrm{rad} / \mathrm{s},
\end{aligned}
$$

e a seguinte matriz de modos massa-normalizados

$$
\boldsymbol{\Phi} \approx\left[\begin{array}{ccccc}
0.274 & -0.353 & 0.167 & -0.353 & 0.382 \\
0.274 & 0.353 & 0.167 & 0.353 & 0.382 \\
0.099 & -0.353 & 0.418 & 0.353 & -0.254 \\
0.099 & 0.353 & 0.418 & -0.353 & -0.254 \\
0.331 & 0.000 & -0.176 & 0.000 & -0.160
\end{array}\right]
$$


cujos valores foram truncados na terceira casa decimal.

Partindo-se da equação (4-17), que representa as equações da dinâmica na forma desacoplada, obteve-se a seguinte formulação do sistema em espaço de estados

$$
\dot{\mathrm{Z}}=\mathrm{AZ}+\mathrm{BU}
$$

onde

$$
\begin{aligned}
& \mathbf{A}=\left[\begin{array}{cccccccccc}
0 & 0 & 0 & 0 & 0 & 1 & 0 & 0 & 0 & 0 \\
0 & 0 & 0 & 0 & 0 & 0 & 1 & 0 & 0 & 0 \\
0 & 0 & 0 & 0 & 0 & 0 & 0 & 1 & 0 & 0 \\
0 & 0 & 0 & 0 & 0 & 0 & 0 & 0 & 1 & 0 \\
0 & 0 & 0 & 0 & 0 & 0 & 0 & 0 & 0 & 1 \\
-\omega_{1}^{2} & 0 & 0 & 0 & 0 & 0 & 0 & 0 & 0 & 0 \\
0 & -\omega_{2}^{2} & 0 & 0 & 0 & 0 & 0 & 0 & 0 & 0 \\
0 & 0 & -\omega_{3}^{2} & 0 & 0 & 0 & 0 & 0 & 0 & 0 \\
0 & 0 & 0 & -\omega_{4}^{2} & 0 & 0 & 0 & 0 & 0 & 0 \\
0 & 0 & 0 & 0 & -\omega_{5}^{2} & 0 & 0 & 0 & 0 & 0
\end{array}\right], \\
& \mathbf{B}=\left[\begin{array}{lllll}
0 & 0 & 0 & 0 & 0 \\
0 & 0 & 0 & 0 & 0 \\
0 & 0 & 0 & 0 & 0 \\
0 & 0 & 0 & 0 & 0 \\
0 & 0 & 0 & 0 & 0
\end{array}\right] \\
& \mathbf{Z}(t)=\left[\begin{array}{c}
y_{1}(t) \\
\vdots \\
y_{5}(t) \\
\dot{y}_{1}(t) \\
\vdots \\
\dot{y}_{5}(t)
\end{array}\right], \quad \mathbf{U}(t)=\left[\begin{array}{c}
0 \\
0 \\
0 \\
0 \\
f(t)
\end{array}\right]
\end{aligned}
$$

Integrando-se numericamente a equação (6-9), utilizando-se o comando 
ode45, com um passo de tempo $\Delta t=10^{-4} \mathrm{~s}$ e C.I's nulas, obteve-se o vetor de estados $\mathbf{Z}(t)$. A partir das suas componentes $y_{i}(t), i=1, \ldots, 5$ obteve-se o vetor de deslocamentos $\mathbf{Y}(t)$, de modo que a resposta do sistema no domínio do tempo foi obtida fazendo-se $\mathbf{X}(t)=\mathbf{\Phi} \mathbf{Y}(t)$. O resultado é dado nos gráficos da figura 6.7. supondo que $m=2 \mathrm{~kg}$ e $k=3 \mathrm{~N} / \mathrm{m}$, para uma amplitude $F_{0}=2$ $N$ e frequência $\omega_{f}=5 \mathrm{rad} / \mathrm{s}$ do forçamento externo.
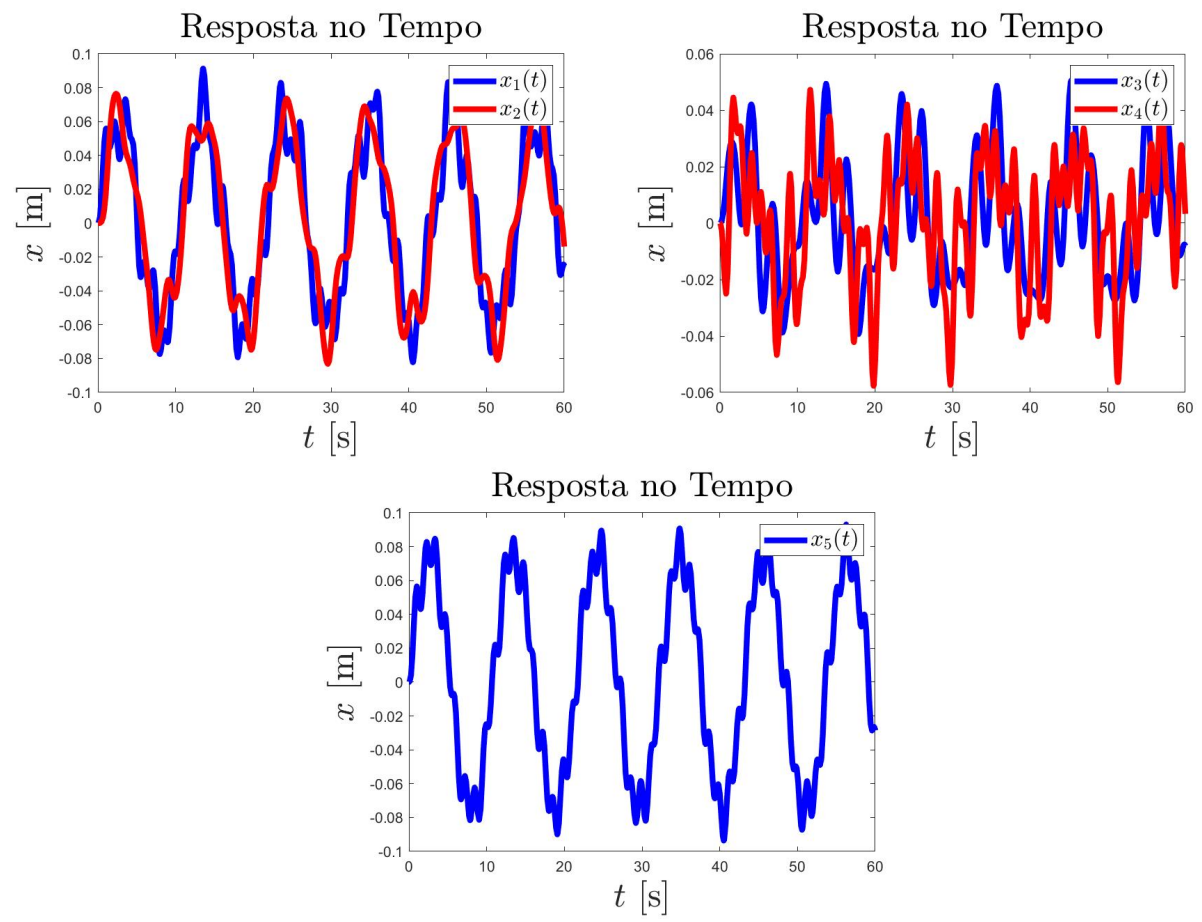

Figura 6.7: resposta no tempo do sistema 2 sem amortecimento, para $\omega_{f}=$ $5 \mathrm{rad} / \mathrm{s}$.

Simulando o sistema 2 com $\omega_{f}=\omega_{1}$, obteve-se os gráficos mostrados na figura 6.8 .

Neste caso, observa-se que o sistema 2 entra em ressonância em todos os seus graus de liberdade, pois as amplitudes de cada um destes passam a crescer linearmente com o tempo, conforme mostra a figura 6.8. O mesmo foi realizado para as demais frequências naturais, observando-se que para $\omega_{f}=\omega_{3}$ e $\omega_{f}=\omega_{5}$, os gráficos obtidos se assemelham ao da figura 6.8, porém o crescimento das amplitudes é mais lento. Para $\omega_{f}=\omega_{2}$ e $\omega_{f}=\omega_{4}$, as respostas não apresentaram o comportamento típico de ressonância.

A resposta do sistema 2 no domínio da frequência, considerando ainda o caso sem amortecimento, foi obtida a partir da equação (4-23), que representa um elemento da matriz de FRF do sistema. Neste caso, como o sistema apresenta cinco graus de liberdade, a matriz de FRF tem dimensão $5 \times 5$, ou seja, ela possui 25 elementos, cada um destes dado por uma função expressa 


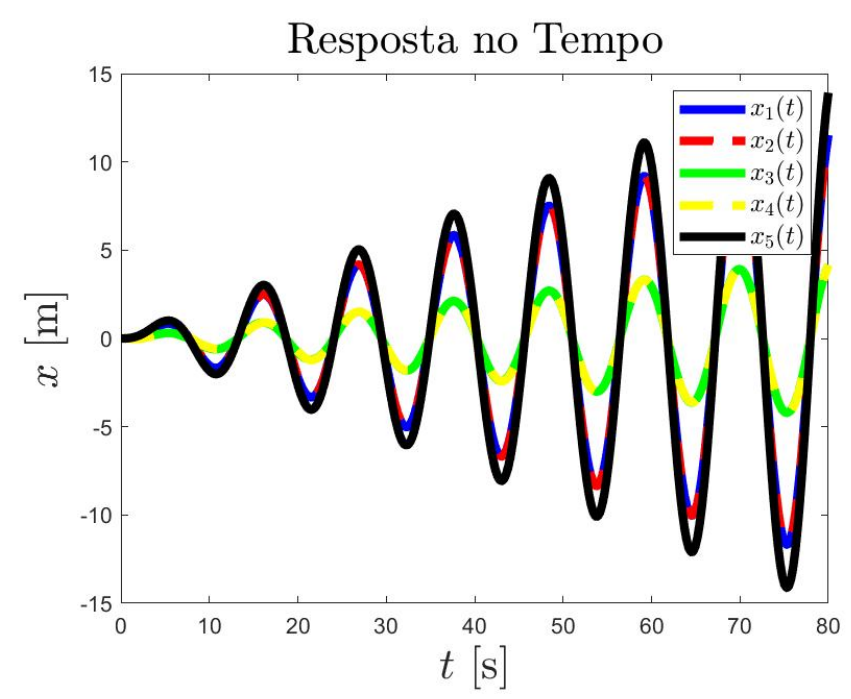

Figura 6.8: resposta no tempo do sistema 2 sem amortecimento, para $\omega_{f}=\omega_{1}$.

pela equação (4-23). Como a forma do gráfico de cada um dos elementos da matriz de FRF é muito semelhante, apenas alguns deles foram obtidos através da simulação numérica, com todos os seus valores dados em escala decibel. A figura 6.9 mostra o gráfico do elemento $h_{11}(\omega)$ da matriz de FRF do sistema 2 sem amortecimento.

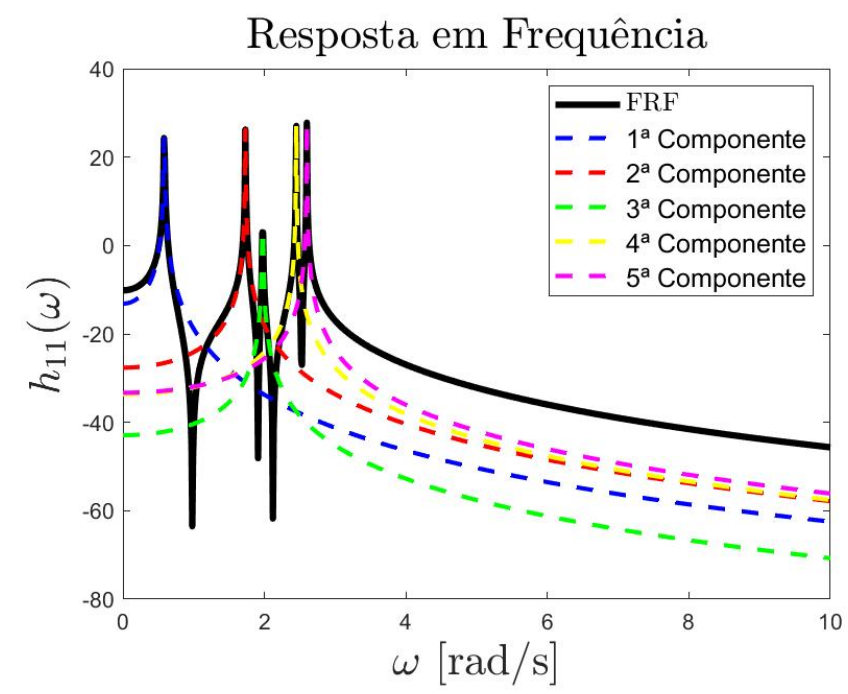

Figura 6.9: resposta em frequência do sistema 2 sem amortecimento.

A curva em preto representa $h_{11}(\omega)$ e as curvas em cores representam cada uma das suas componentes, ou seja, os termos que se somam na equação (4-23). De acordo com [3], cada um dos picos do gráfico de $h_{11}(\omega)$ está relacionado a uma frequência natural do sistema. No caso, como o sistema apresenta cinco graus de liberdade, existirão cinco frequências naturais e, como todas elas neste 
caso são distintas, devem existir cinco picos no gráfico de $h_{11}(\omega)$. Desta forma, o resultado obtido está de acordo com o esperado, pois o gráfico de $h_{11}(\omega)$ apresenta cinco pontos de máximo. Porém, vale ressaltar que, no gráfico obtido, por conta da discretização dos valores das frequências, os picos mostrados na figura 6.9 se localizam em valores que são próximos das frequências naturais, de modo que, a ressonância não ocorre de fato. Porém, o que o gráfico indica é que a medida que $\omega_{f}$ se aproxima de determinada frequência natural $\omega_{n}$, o valor da FRF tende a ser cada vez maior. No momento em que $\omega_{f}$ é igual a uma das frequências naturais, o valor da FRF é infinito e, como comentado anteriormente, não pode ser representado de forma gráfica.

Desta forma, observa-se que, em um sistema com $n$ graus de liberdade existirão no máximo $n$ formas de se colocá-lo para vibrar de forma a se obter o fenômeno de ressonância, basta que o forçamento externo seja aplicado com uma das frequências naturais do sistema.

O gráfico dado na figura 6.9 representa o comportamento do sistema relativo ao grau de liberdade $x_{1}(t)$, quando uma força externa é aplicado sobre ele neste mesmo grau de liberdade. No caso, se uma força externa for aplicada no sistema no grau de liberdade $x_{3}(t)$ e quiser se conhecer a resposta referente ao grau de liberdade $x_{2}(t)$, por exemplo, deve-se obter a FRF $h_{23}(\omega)$. Este caso também foi simulado numericamente no MatLab, e o resultado é mostrado na figura 6.10 .

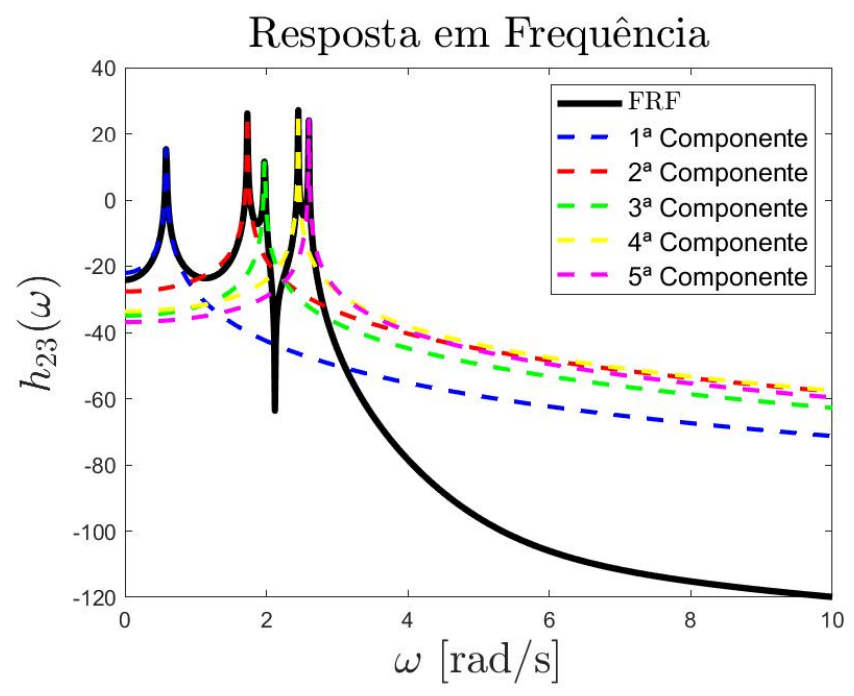

Figura 6.10: resposta em frequência do sistema 2 sem amortecimento.

O resultado obtido é semelhante ao da figura 6.9 e tem a mesma interpretação.

Simulando o sistema 2, mas considerando que o forçamento externo está agindo na maior massa, como é mostrado na figura 5.2 , observou-se que as 
FRF's $h_{i 5}(\omega), i=1, \ldots, 5$ apresentaram somente três "picos de ressonância", referentes as frequências naturais $\omega_{1}, \omega_{3}$ e $\omega_{5}$. Este resultado está de acordo com o resultado que foi obtido no domínio do tempo, pois conforme observado, o sistema não entrou em ressonância quando o forçamento externo, aplicado à maior massa, estava nas frequências $\omega_{2}$ ou $\omega_{4}$. Este fato se deve aos elementos $\phi_{52}$ e $\phi_{54}$ na equação (4-23). Como estes elementos na matriz de modos massanormalizados $\boldsymbol{\Phi}$ são nulos (os valores obtidos através do MatLab são da ordem de $\left.10^{-10}\right)$, a segunda e a quarta componentes das FRF's $h_{i 5}(\omega)$ são anuladas, de forma que o sistema não entra em ressonância quando $\omega_{f}=\omega_{2}$ ou $\omega_{f}=\omega_{4}$, fazendo com que só tenha três picos nas FRF's $h_{i 5}(\omega)$.

Na figura 6.11 é mostrado o gráfico da $\operatorname{FRF} h_{15}(\omega)$, que representa a relação entre a amplitude do forçamento externo aplicado na maior massa do sistema e a amplitude da resposta em regime permanente referente ao grau de liberdade $x_{1}(t)$.

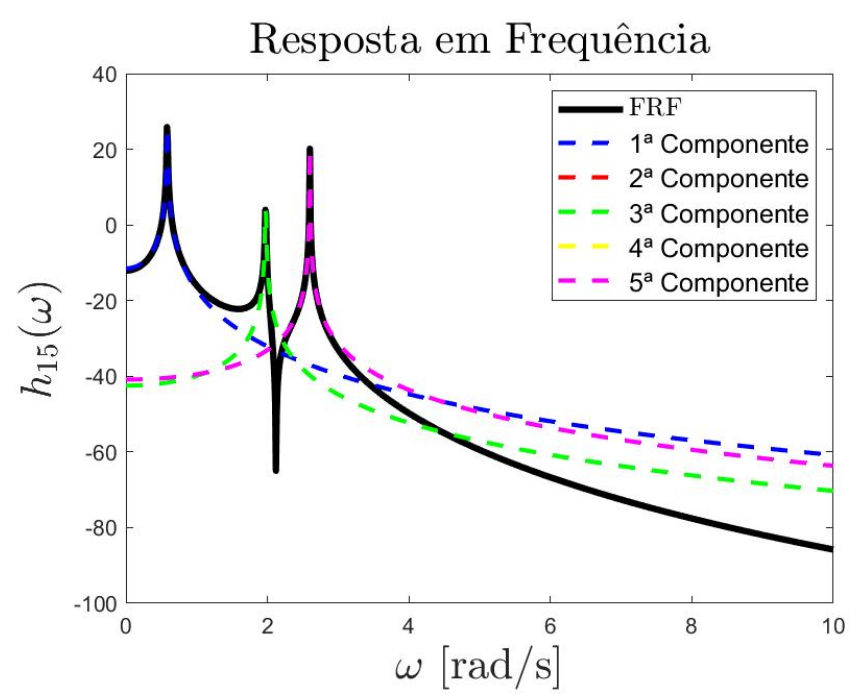

Figura 6.11: resposta em frequência do sistema 2 sem amortecimento.

Conforme verificado, os valores da segunda e da quarta componentes de $h_{15}(\omega)$ valem $-\infty$ (um valor negativo, mas com valor absoluto muito elevado) em escala decibel, o que representa valores nulos na escala original, indicando que, de fato, a amplitude da resposta do sistema no grau de liberdade $x_{1}(t)$ é muito pequena quando o forçamento externo é aplicado na maior massa do sistema nas frequências $\omega_{2}$ ou $\omega_{4}$.

\section{3}

\section{Sistema 2 com Amortecimento}

Considerando ainda o sistema 2, mas levando em conta o amortecimento, verificou-se que a matriz de amortecimento $\mathbf{C}$ é não proporcional. Portanto, o 
sistema de e.d.o's (4-24) não poderá ser desacoplado. Para se obter a resposta do sistema no domínio do tempo, utilizou-se novamente a formulação em espaço de estados, mas considerando o efeito do amortecimento. Desta forma, obtevese a seguinte equação matricial

$$
\dot{\mathrm{Z}}=\mathrm{AZ}+\mathrm{BU}
$$

onde

$$
\begin{aligned}
& \mathbf{A}=\left[\begin{array}{cccccccccc}
0 & 0 & 0 & 0 & 0 & 1 & 0 & 0 & 0 & 0 \\
0 & 0 & 0 & 0 & 0 & 0 & 1 & 0 & 0 & 0 \\
0 & 0 & 0 & 0 & 0 & 0 & 0 & 1 & 0 & 0 \\
0 & 0 & 0 & 0 & 0 & 0 & 0 & 0 & 1 & 0 \\
0 & 0 & 0 & 0 & 0 & 0 & 0 & 0 & 0 & 1 \\
\frac{-3 k}{m} & 0 & \frac{k}{m} & 0 & \frac{2 k}{m} & -\frac{2 c}{m} & 0 & \frac{c}{m} & 0 & \frac{c}{m} \\
0 & -\frac{3 k}{m} & 0 & \frac{k}{m} & 2 k / m & 0 & -\frac{2 c}{m} & 0 & c / m & c / m \\
\frac{k}{m} & 0 & -\frac{3 k}{m} & 0 & 0 & \frac{c}{m} & 0 & -\frac{2 c}{m} & 0 & 0 \\
0 & \frac{k}{m} & 0 & -\frac{3 k}{m} & 0 & 0 & \frac{c}{m} & 0 & -\frac{2 c}{m} & 0 \\
2 k / 3 m & 2 k / 3 m & 0 & 0 & -\frac{4 k}{3 m} & \frac{c}{3 m} & \frac{c}{3 m} & 0 & 0 & -2 c / 3 m
\end{array}\right] \\
& \mathbf{B}=\left[\begin{array}{ccccc}
0 & 0 & 0 & 0 & 0 \\
0 & 0 & 0 & 0 & 0 \\
0 & 0 & 0 & 0 & 0 \\
0 & 0 & 0 & 0 & 0 \\
0 & 0 & 0 & 0 & 0 \\
0 & 0 & 0 & 0 & 0 \\
0 & 0 & 0 & 0 & 0 \\
0 & 0 & 0 & 0 & 0 \\
0 & 0 & 0 & 0 & 0 \\
0 & 0 & 0 & 0 & \frac{1}{3 m}
\end{array}\right], \\
& \mathbf{Z}(t)=\left[\begin{array}{c}
x_{1}(t) \\
\vdots \\
x_{5}(t) \\
\dot{x}_{1}(t) \\
\vdots \\
\dot{x}_{5}(t)
\end{array}\right], \quad \mathbf{U}(t)=\left[\begin{array}{c}
0 \\
0 \\
0 \\
0 \\
f(t)
\end{array}\right]
\end{aligned}
$$


Integrando-se numericamente a equação (6-13), utilizando-se o comando ode45, com um passo de tempo $\Delta t=10^{-4} \mathrm{~s}$ e C.I's nulas, obteve-se o vetor de estados $\mathbf{Z}(t)$. A partir das suas componentes $x_{i}(t), i=1, \ldots, 5$ obtevese o vetor de deslocamentos $\mathbf{X}(t)$, que fornece a resposta do sistema $2 \mathrm{com}$ amortecimento no domínio do tempo. Os gráficos obtidos para cada um dos graus de liberdade são dados na figura 6.12 .
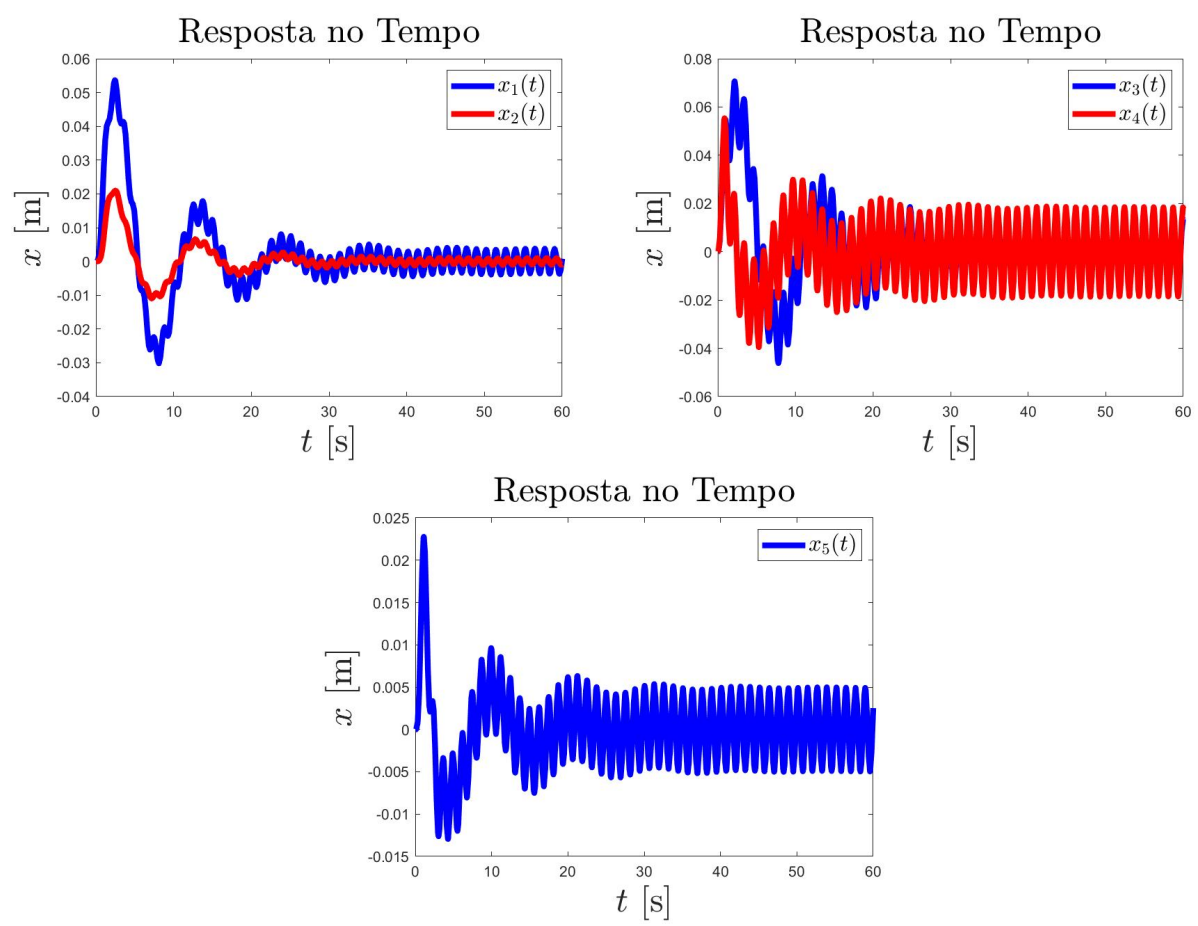

Figura 6.12: resposta no tempo do sistema 2 com amortecimento para $\omega_{f}=$ $5 \mathrm{rad} / \mathrm{s}$.

Na simulação realizada, foi considerado um coeficiente de amortecimento linear viscoso $c=2.5 \mathrm{~N} \cdot \mathrm{s} / \mathrm{m}$. Comparando os gráficos das figuras 6.12 e 6.7 , observa-se que, após o sistema entrar em regime permanente, as amplitudes das respostas no domínio do tempo, para o caso com amortecimento, são menores do que para o caso sem amortecimento, mostrando o efeito da dissipação de energia no sistema.

A resposta em frequência do sistema 2 com amortecimento foi obtida a partir da determinação das frequências naturais e modos complexos. Resolvendo-se o problema de autovalor complexo, dado na equação (4-34), obteve-se os autovalores $s_{r}$ e os autovetores associados $\boldsymbol{\theta}_{r}$. A partir da relação dada na equação (4-36), os autovetores $\boldsymbol{\xi}_{r}$ foram determinados e, em seguida, agrupados em colunas em uma matriz $\boldsymbol{\Xi}$. O resultado obtido é dado abaixo

$$
s_{1} \approx-0.109-0.578 i
$$




$$
\begin{aligned}
& s_{3} \approx-1.932-1.649 i, \\
& s_{5} \approx-1.875-1.576 i, \\
& s_{7} \approx-0.624-1.615 i, \\
& s_{9} \approx-0.874-1.803 i, \\
& \Xi \approx\left[\begin{array}{ccccc}
-0.513-0.317 i & 0.295-0.207 i & 0.309-0.229 i & 0.278-0.380 i & -0.098+0.219 i \\
-0.513-0.317 i & 0.295-0.207 i & -0.309+0.229 i & -0.278+0.380 i & -0.098+0.219 i \\
-0.209-0.086 i & -0.256+0.232 i & -0.309+0.229 i & 0.278-0.380 i & -0.269+0.300 i \\
-0.209-0.086 i & -0.256+0.232 i & 0.309-0.229 i & -0.278+0.380 i & -0.269+0.300 i \\
-0.632-0.367 i & -0.099+0.006 i & 0.000-0.000 & 0.000+0.000 & 0.108-0.203 i
\end{array}\right]
\end{aligned}
$$

cujos valores foram truncados na terceira casa decimal. Vale ressaltar que os autovalores e autovetores são dados em pares de complexos conjugados. Portanto, os resultados mostrados acima se referem a apenas um dos elementos de cada par.

Foram obtidos gráficos das FRF's a partir da equação (4-53). O resultado é dado nas figuras 6.13 e 6.14 .

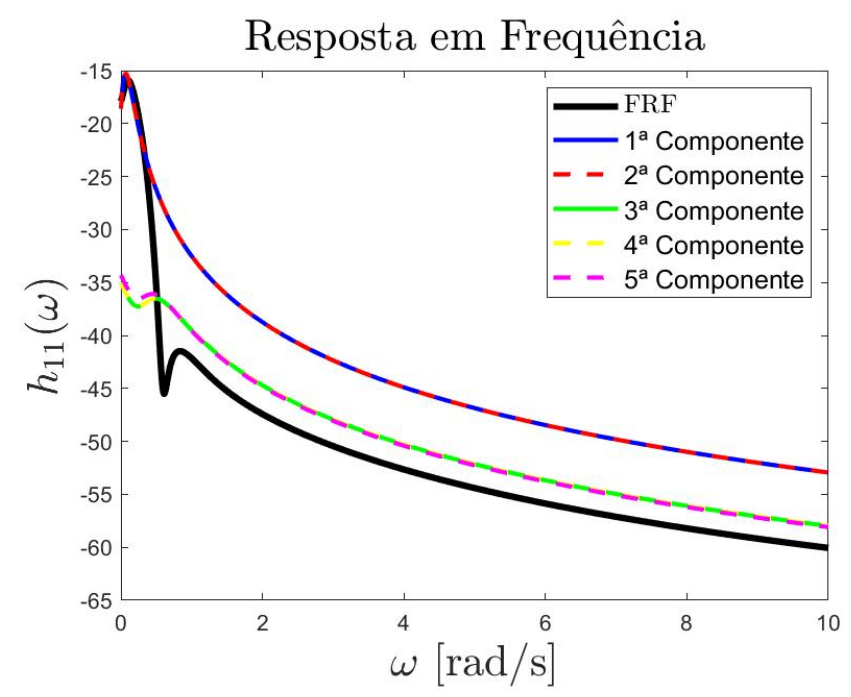

Figura 6.13: resposta em frequência do sistema 2 com amortecimento.

onde cada uma das componentes das FRF's são dadas pela soma de dois termos na equação (4-53). Conforme se observa, o gráfico das FRF's apresenta valores negativos, e estes tendem a diminuir cada vez mais com o aumento da frequência do forçamento externo. Desta forma, como os valores dados nos gráficos estão em escala decibel, os valores das FRF's estão muito próximos de 


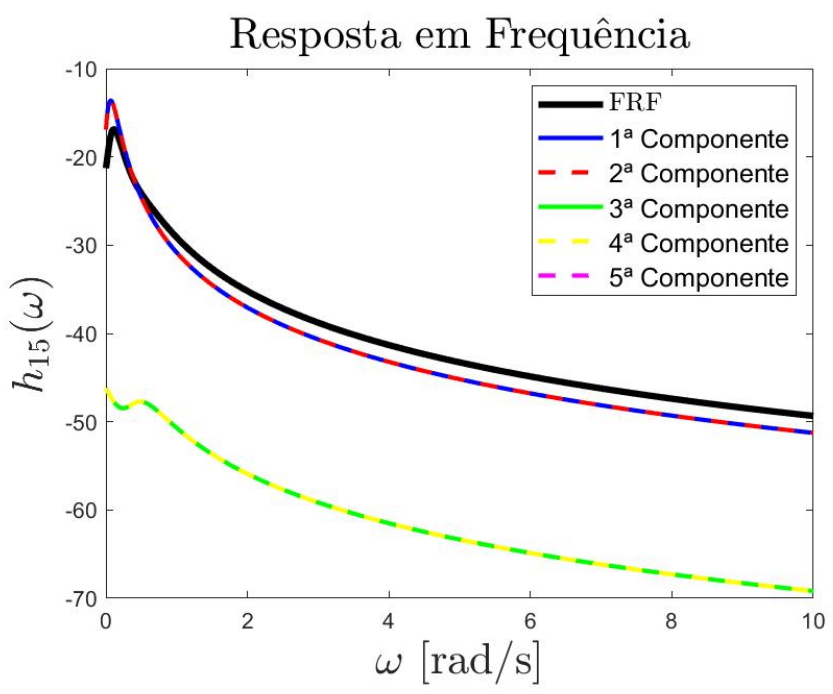

Figura 6.14: resposta em frequência do sistema 2 com amortecimento.

zero na escala original e se aproximam cada vez mais deste valor a medida em que a frequência do forçamento externo aumenta. Isto mostra que as FRF's apresentam um comportamento semelhante a FRF de um sistema de um grau de liberdade, dado na figura 6.2, cujos valores se aproximam cada vez mais de zero a medida em que a frequência aumenta. Portanto, através do resultado obtido, verifica-se o efeito da dissipação de energia no sistema, pois como a FRF representa a razão entre amplitude da resposta em regime permanente e a amplitude do forçamento externo, têm-se que a amplitude da resposta se aproxima cada vez mais de zero com o aumento da frequência.

Além disto, diferentemente do caso sem amortecimento, as componentes individuais das FRF's não mostram uma contribuição específica nos valores das frequências naturais, justamente por estas assumirem valores complexos em sistemas com amortecimento não proporcional. 


\section{Conclusão}

A partir do trabalho desenvolvido, foi possível aprofundar e ampliar a teoria vista do curso de vibrações mecânicas da PUC-Rio, através do estudo da bibliografia e do desenvolvimento de exemplos que ilustrem o uso dos conceitos aprendidos.

O sistema mecânico com um grau de liberdade serviu como base para revisar conceitos fundamentais de vibrações, entre eles o de ressonância, que posteriormente pode ser agregado as interpretações feitas nos gráficos do sistema com múltiplos graus de liberdade. A teoria de análise modal apresentada possibilitou determinar alguns termos da matriz de FRF e, através destes, pode-se mostrar a influência das frequências naturais e modos de vibração no comportamento dinâmico dos sistemas com múltiplos graus de liberdade, com e sem amortecimento.

No caso sem amortecimento, pode-se entender como que os modos normais afetam a dinâmica do sistema, pois estes contribuem para a ocorrência da ressonância nos valores das frequências naturais. No caso com amortecimento linear viscoso, a contribuição dos modos complexos não pode ser observada, pois, neste caso, como as frequências naturais e as componentes dos modos tem valores complexos, as parcelas individuais das FRF's não indicam uma influência direta destes parâmetros modais na dinâmica do sistema, pois os gráficos das parcelas individuais não coincidem com o gráfico da FRF, como ocorre no caso sem amortecimento, nas regiões próximas das frequências naturais.

Outro ponto importante foi a não ocorrência de ressonância em algumas frequências naturais do sistema 2 sem amortecimento. Isto foi devido ao fato de dois modos normais apresentarem pelo menos uma componente nula. Desta forma, a contribuição destes dois modos para determinadas FRF's são anuladas, eliminando a influência destes modos no comportamento do sistema, conforme foi observado na figura 6.11 .

Portanto, o trabalho desenvolvido conseguiu ilustrar, através de alguns exemplos simples, o procedimento da decomposição modal e, através deste, permitir entender como que a dinâmica de um sistema com múltiplos graus de liberdade é decomposta pelos parâmetros modais: frequências naturais e modos de vibração. E a partir disto, conseguir entender como que o sistema se comporta sob a ação de esforços externos, com foco no fenômeno da ressonância. 


\section{Referências Bibliográficas}

[1] HE, J., FU, Z. F. Modal Analysis. Butterworth Heinemann, Oxford, 2001.

[2] INMAN, D. Engineering Vibrations, fourth edition. Pearson, New Jersey:, 2014.

[3] SAmpaio, R., WAGNER, G. Apostila de Análise Modal. Rio de Janeiro, 2016.

[4] LIMA, R. Notas de Aula do Curso de Vibrações Mecânicas da PUC-Rio.

[5] ADHIKARI, S. Vibration of Damped Systems. Department of Aerospace Engineering, Bristol.

[6] LI, L., HU, Y., WANG, X., LU., L. A hybrid expansion method for frequency response functions of non-proportionally damped systems. Mechanical Systems and Signal Processing, 42:31-41, 2014.

[7] AVITABILE, P. Modal Testing A Practitioner's Guide. WILEY, Estados Unidos, 2018.

[8] P. AVITABILE. Experimental modal analysis: A simple non-mathematical presentation. Sound and Vibration, 35:20-31, 2001.

[9] SAmpaio, R., CATAldo, E., BRAnd ÃO, A. Análise e Processamento de Sinais, Notas em Matemática Aplicada, volume 22. SBMAC, São Carlos, São Paulo, Brasil, 2012. 